R. FRANK

CERMES (ENPC-LCPC)

Cité Descartes

Champs-sur-Marne

77455 Marne-la-Vallée

Cedex 2

\section{J.-B. KOVARIK}

Port Autonome de Rouen 34 , bd de Boisguilbert 76022 Rouen Cedex 3

\title{
Comparaison des niveaux de sécurité, calage d'un coefficient de modèle pour la résistance ultime des pieux sous charges axiales
}

\author{
- 0 Les changements intervenus depuis l'époque de la pré- \\ norme ENV 1997-1 et la nécessité actuelle d'étalonner les \\ raccordements nationaux à l'Eurocode 7 (norme EN 1997 - \\ 1), justifient la mise au point de méthodes opérationnelles \\ de comparaison des niveaux de sécurité et de calage des \\ coefficients partiels. Pour le dimensionnement des pieux \\ sous charges axiales de compression vis-à-vis de l'état \\ limite de défaut de capacité portante, on met en évidence \\ que les niveaux globaux de sécurité recommandés par la \\ norme européenne EN 1997-1 et par le règlement officiel \\ français « Fascicule $n^{\circ} 62$ - Titre V ) présentent des \\ ordres de grandeur comparables. Un calage par \\ ajustement est tout d'abord proposé et permet de \\ déboucher sur une évaluation du coefficient de modèle de \\ résistance $\gamma_{\mathrm{Rd}}$ qui, utilisé conjointement avec les valeurs \\ des coefficients partiels recommandées pour l'approche 2 \\ de l'EN 1997-1, permet de retrouver les niveaux de \\ sécurité du Fascicule 62-V. Pour le calcul à partir des \\ résultats des essais de sol, un calage probabiliste des \\ coefficients partiels de résistance est également mis en \\ œuvre à l'aide d'une série d'essais de pieux; on ouvre un \\ débat sur les niveaux de sécurité à prendre en compte dans \\ la combinaison fondamentale: la méthode des « valeurs de \\ calcul ») de l'annexe $\mathrm{C}$ de l'Eurocode: Bases du calcul des \\ structures (norme EN 1990) donnerait des résultats trop \\ sécuritaires au regard de la pratique; on met en œuvre une \\ ( méthode inverse ) qui remonte à une probabilité cible de \\ ruine sous-jacente, en référence aux coefficients partiels \\ proposés par les codes de calcul étudiés. Les résultats des \\ calages des coefficients partiels sont des intervalles du fait \\ des diverses interprétations envisageables de la valeur \\ caractéristique des propriétés des sols. Ces résultats \\ pourront être réexaminés pour tenir compte de nouvelles \\ comparaisons réalisées entre le modèle pressiométrique \\ (ou un autre modèle à partir d'essais de sol) et les essais \\ de chargement statique de pieux.
}

Mots clés : pieu, capacité portante, Eurocode 7, sécurité, coefficients partiels, calage, probabilité.

\section{Comparison of safety levels, calibration of a model factor for the bearing resistance of piles under axial loads}


standard EN 1997-1 and by the French official design code ( Fascicule $n^{\circ} 62$ - Titre $V$ ) have comparable magnitudes. An adjusting method is first proposed and allows the assessment of the resistance model factor $\gamma_{\mathrm{Rd}}$ which, used together with the values of the partial factors recommended in design approach 2 of EN 1997-1, yields the same safety levels as Fascicule 62-V. For the calculation from ground test results, a probabilistic calibration of the partial resistance is also performed by means of a series of pile tests; the question of the safety levels to take into account with the fundamental combination is debated: the use of the (design values» method of Annex $C$ of Eurocode: Basis of design (standard EN 1990) would give excessively safe results when compared to common practice; an (cinverse method $)$ is used which goes back to a subjacent target probability of failure, referring to partial factors proposed in the reviewed codes of practice. The results of the calibrations are ranges of values because of the various possible interpretations of the characteristic value of ground properties. It will be possible to revise the results to take into account new comparisons performed between the pressuremeter model (or any other model from ground test results) and the static load test on piles.

Key words : pile, bearing resistance, Eurocode 7, safety, partial factors, resistance model factor, calibration, probability.

Deux méthodes de calage des coefficients partiels de résistance sont mises en œuvre pour l'approche 2 des Eurocodes (en supposant des charges sur le pieu uniquement d'origine non géotechnique), avec application numérique: le calage par ajustement et le calage probabiliste. Pour ce dernier, on exploite l'information provenant d'une série d'essais de chargement statique de pieux comparant les prévisions du modèle pressiométrique à la réalité mesurée de la résistance des pieux, et l'on donne à la fin le formulaire complet de la méthode, permettant d'exécuter le cas échéant de nouveaux calculs avec un autre jeu de données, notamment avec la comparaison d'un nouveau modèle pressiométrique aux essais de chargement statique de pieux, actuellement en cours au LCPC (Bustamante et Gianeselli, 2003).

Cet article constitue une extension de la comparaison qui avait été effectuée par Frank (1997) à l'époque de la pré-norme pour l’EN 1997-1 de 1994 (ENV 1997-1, 1994, voir AFNOR 1996).

\section{Définition des valeurs représentatives Relations de base} déré comme un cas particulier. Par ailleurs, il faut garder présent à l'esprit que ces comparaisons ne concernent pas la sécurité globale de la fondation sur pieux, où interviennent d'autres éléments que l'état limite de défaut de capacité portante axiale du pieu isolé (et le modèle de calcul qui lui est associé): citons par exemple l'effet de groupe ainsi que les états limites de service (ELS), qui sont d'ailleurs traités très différemment dans l'EN 1997-1 et dans le Fascicule n 62 Titre V.

Dans la perspective de devoir effectuer le calage des niveaux de sécurité dans le cadre des normes nationales d'application complémentaires à l'Eurocode 7, on commence par comparer globalement le niveau de sécurité recommandé par l'EN 1997-1 à celui préconisé par la pratique nationale française.

\section{Valeurs représentatives de la résistance}

Pour un pieu chargé en compression, les relations de base pour la justification vis-à-vis de l'état limite de défaut de la capacité portante peuvent être résumées de la manière suivante. On utilise la terminologie et les notations de l'EN 1997-1 (CEN, 2004):

$R_{c}$ représente la résistance en compression (compressive resistance, capacité portante ultime en compression):

$R_{c, k}$ est la valeur caractéristique de cette résistance, $R_{c, d}$ est la valeur de calcul de cette résistance. 


\section{2}

\section{Valeur caractéristique de la résistance}

Les coefficients de réduction statistique $\xi$ introduits ci-après ont pour objet de " gérer » l'incertitude liée à la variabilité spatiale de la résistance du pieu (Bauduin, 2001). Avec les règles de l'EN 1997-1, lorsque cette variabilité spatiale est inférieure à $10-12 \%$, la valeur caractéristique est déterminée par la moyenne des résistances; dans le cas contraire, elle est déterminée par la plus petite des résistances. Plus le nombre d'essais augmente, plus la valeur de $\xi$ diminue.

\subsection{1}

\section{Calcul sur la base d'essais de chargement statique de pieux}

Selon l’EN 1997-1:

$$
\mathrm{R}_{\mathrm{c}, \mathrm{k}}=\operatorname{Inf}\left\{\mathrm{R}_{\mathrm{c}, \mathrm{m}} / \xi_{1} \mathrm{R}_{\mathrm{c}, \min } / \xi_{2}\right.
$$

où: $R_{c, m}$ est la valeur moyenne des résistances mesurées,

$R_{c, \min }$ est la plus petite des résistances mesurées, $\xi_{1}$ et $\xi_{2}$ sont des coefficients de réduction statistique dépendant du nombre $\mathrm{N}$ d'essais de chargement statique de pieux effectués, donnés dans le tableau I.

Dans le cas du Fascicule $n^{\circ} 62$ - Titre V:

- si un seul essai de chargement statique de pieu est effectué:

$$
\mathrm{R}_{\mathrm{c}, \mathrm{k}}=\mathrm{R}_{\mathrm{c}, \mathrm{mes}} / \xi
$$

où $\xi=1,20$;

- si plusieurs essais de chargement statique sont effectués, la valeur caractéristique à retenir est déterminée en principe directement à partir d'une évaluation critique des résultats des essais. Cependant, lorsque l'estimation du coefficient de variation, ou dispersion (écart type/moyenne) n'atteint pas $30 \%$, on peut utiliser la relation suivante (et c'est ce qui est fait dans la suite de cet article):

$$
\mathrm{R}_{\mathrm{c}, \mathrm{k}}=\mathrm{R}_{\mathrm{c}, \min } \cdot\left(\mathrm{R}_{\mathrm{c}, \min } / \mathrm{R}_{\mathrm{c}, \max }\right)^{\mathrm{s}^{\prime}}
$$

où: $R_{c \text { min }}$ et $R_{c \text { max }}$ sont les valeurs minimale et maximale mesurées, $\xi^{\prime}$ est donné dans le Tableau II.

\subsubsection{2.}

\section{Calcul sur la base d'essais de sol}

\subsubsection{Méthode des ( pieux modèle 》)}

Selon l'EN 1997-1, quand les résultats d'essais exécutés sur le sol sont utilisés, on doit calculer une valeur de la résistance $R_{c, \text { cal }}$ pour chaque profil d'essais de sol. Ces valeurs de $R_{c, \text { cal }}$ sont en quelque sorte des valeurs de ( pieux modèle »).

La valeur caractéristique de la résistance est alors déterminée comme suit:

$$
\mathrm{R}_{\mathrm{c}, \mathrm{k}}=\operatorname{Inf}\left\langle\mathrm{R}_{\mathrm{c}, \text { cal, } \mathrm{m}} / \xi_{3} \mathrm{R}_{\mathrm{c}, \text { cal } \min } / \xi_{4}\right.
$$

où: $R_{\text {cal }}$ est la moyenne des valeurs de la résistance telle qu'elles sont calculées à partir des valeurs des propriétés des sols prises en considération dans le modèle de résistance (ou méthode de prévision); $R_{c, c a l \min }$ est la plus petite des valeurs de la résistance ainsi calculées,

$\xi_{3}$ et $\xi_{4}$ sont des coefficients de réduction statistique dépendant du nombre $\mathrm{N}$ d'essais de sol réalisés (nombre de profils) donnés dans le Tableau III.

TABLEAU II Fascicule $n^{\circ} 62$ - Titre V - Coefficients $\xi^{\prime}$. Fascicule $n^{\circ} 62$ - Titre V. $\xi^{\prime}$ factors

\begin{tabular}{cccccc}
\hline Nombre d'essais de chargement & 2 & 3 & 4 & 5 \\
\hline$\xi^{\prime}$ & 0,55 & 0,20 & 0,07 & 0,00
\end{tabular}

TABLEAU EN 1997-1. Coefficients de réduction statistique $\xi$ pour le calcul de $R_{c, k}$ à partir d'essais de chargement statique de pieux ( $\mathrm{N}$ : nombre d'essais de chargement statique).

EN 1997-1. Statistical reduction factor $\xi$ to derive $R_{c, k}$ from pile static load tests ( $N$ : number of static load tests).

$\begin{array}{llllll}\xi \text { pour } \mathrm{N}= & 1 & 2 & 3 & 4 & \geq 5\end{array}$

\begin{tabular}{llllll} 
a) Coefficient $\xi_{1}$ sur la moyenne $\mathrm{R}_{c, m}$ & 1,40 & 1,30 & 1,20 & 1,10 & 1,00 \\
b) Coefficient $\xi_{2}$ sur la valeur de plus basse $\mathrm{R}_{c, \min }$ & 1,40 & 1,20 & 1,05 & 1,00 & 1,00 \\
\hline
\end{tabular}

Note: lorsque les charges peuvent se reporter des pieux « faibles » vers les pieux « forts », l'EN 1997 autorise de diviser éventuellement ces coefficients par 1,1 .

TABLEAU III EN 1997-1. Coefficients de réduction statistique $\xi$ dans le cas d'un modèle de prévision à partir de résultats d'essais de sol ( $\mathrm{N}$ : nombre de profils d'essais de sol).

EN 1997-1. Statistical reduction factor $\xi$ in the case of a prediction model from ground test results ( $N$ : number of profile of tests).

\begin{tabular}{lrrrrrrr}
\multicolumn{1}{c}{$\xi$ pour $\mathrm{N}=$} & 1 & 2 & 3 & 4 & 5 & 7 & 10 \\
\hline a) Coefficient $\xi_{3}$ sur la moyenne $R_{c, \text { cal m }}$ a) $R_{c, \text { cal min }}$ & 1,40 & 1,35 & 1,33 & 1,31 & 1,29 & 1,27 & 1,25 \\
a) Coefficient $\xi_{4}$ sur la valeur la plus basse & 1,27 & 1,23 & 1,20 & 1,15 & 1,12 & 1,08 \\
\hline
\end{tabular}

Note: lorsque les charges peuvent se reporter des pieux « faibles » vers les pieux « forts », l’EN 1997 autorise de diviser éventuellement ces coefficients par 1,1. 


\subsubsection{Méthode ( alternative » ou « directe »}

L'EN 1997-1 prévoit une méthode « alternative » à l'utilisation de la relation (4) et des coefficients $\xi_{3}$ et $\xi_{4}$ pour déterminer $R_{c, k}$, que les auteurs jugent intéressante. En effet, il autorise de déterminer directement les valeurs caractéristiques de la résistance de pointe $\mathrm{q}_{\mathrm{b}, \mathrm{k}}$ et du frottement latéral $\mathrm{q}_{\mathrm{s}, \mathrm{k}}$ à partir de valeurs des paramètres de sol (clause 7.6.2.3 (8)). Il y a donc lieu de considérer que dans ce cas $\xi$ n'est pas donné de façon apparente. Ceci montre notamment que l'on ne fait pas dépendre directement et de façon explicite la résistance calculée du nombre d'essais exécutés sur le sol: en effet c'est au niveau des valeurs caractéristiques ellesmêmes (de la résistance de pointe et du frottement latéral) qu'est prise en compte l'incertitude statistique d'estimation à partir d'un nombre limité d'essais de sol.

Le Fascicule 62-V donne deux modèles de calcul semi-empiriques à partir de résultats d'essais in situ: une modèle à partir des essais au pressiomètre Ménard (MPT) et un modèle à partir des essais de pénétration statique (CPT). Ces deux modèles ont été établis à partir de corrélations avec de nombreux essais de chargement statique de pieux, proposées à l'origine par Bustamante et Gianeselli (1981). Il faut également relever que les essais statiques de pieux sont exécutés en France d'une manière tout à fait conforme à celle de l'ISSMFE (1985) recommandée par l’EN 1997-1.

Le Fascicule 62-V prévoit ainsi de déterminer directement les valeurs caractéristiques de la résistance de pointe $q_{b,}$ et du frottement latéral $q_{s, k}$ à partir de valeurs des propriétés pressiométriques ou pénétrométriques du sol.

\subsubsection{Valeur caractéristique ou valeur moyenne} des propriétés des sols?

L'EN 1997-1 est muet sur la ( nature ») des valeurs représentatives des paramètres de sol à utiliser tant dans la méthode des pieux modèle que dans la méthode (c directe ».

Les valeurs des paramètres de sol utilisés pour déterminer les valeurs caractéristiques de la résistance de pointe dans la méthode " directe » du Fascicule 62$\mathrm{V}$, sont selon l'usage proches des valeurs moyennes et elles incluent, le cas échéant, un élément de sécurité dans leur estimation. Selon les auteurs, la sécurité liée habituellement à la notion de valeur caractéristique est " cachée » dans les relations elles-mêmes, données par le fascicule, entre les propriétés pressiométriques (pressions limites) ou pénétrométriques (résistances de cône) et les paramètres $q_{b, k}$ et $q_{s, k}$. Les auteurs proposent d'appliquer un raisonnement analogue pour les propriétés des sols à utiliser avec l’EN 1997-1.

\section{Valeur de calcul de la résistance}

La valeur de calcul de la résistance $R_{c, d}$ est déterminée par un coefficient partiel, noté de la façon la plus générale $\gamma_{R}$, appliqué à la valeur caractéristique $R_{c, k}$ :

$$
\mathrm{R}_{\mathrm{c}, \mathrm{d}}=\mathrm{R}_{\mathrm{c}, \mathrm{k}} / \gamma_{\mathrm{R}}
$$

Le texte de l'EN 1997-1 utilise toutefois les notations particulières suivantes, détaillant $\gamma_{\mathrm{R}}$ ci-dessus :

$$
\begin{gathered}
\mathrm{R}_{c, d}=\mathrm{R}_{c, \mathrm{k}} / \gamma_{\mathrm{t}} \\
\mathrm{R}_{\mathrm{c}, \mathrm{d}}=\mathrm{R}_{\mathrm{b}, \mathrm{k}} / \gamma_{\mathrm{b}}+\mathrm{R}_{\mathrm{s}, \mathrm{k}} / \gamma_{\mathrm{s}}
\end{gathered}
$$

où: $R_{b, k}$ est la valeur caractéristique de la résistance de pointe;

$\mathrm{R}_{\mathrm{s}, \mathrm{k}}$ est la valeur caractéristique de la résistance de fût (frottement latéral);

$\gamma_{1}, \gamma_{b}$ et $\gamma_{s}$ sont les coefficients partiels sur la résistance totale ou sur les résistances de pointe et de fût.

\section{4}

\section{Valeurs représentatives des actions}

F représente les actions de compression appliquées en tête du pieu:

$\mathrm{F}_{c, k}$ est la valeur caractéristique des actions;

$F_{c, d}$ est la valeur de calcul des actions.

Pour vérifier les états limites ultimes de résistance, les valeurs caractéristiques des actions appliquées $\mathrm{F}_{\mathrm{c}}$ sont multipliées par les coefficients $\gamma_{\mathrm{F}}$ de manière à obtenir leur valeur de calcul:

$$
\mathrm{F}_{c, d}=\gamma_{\mathrm{F}} \cdot \mathrm{F}_{c, k}
$$

\section{5}

\section{Valeurs des coefficients partiels $\gamma$}

Le tableau IV donne les coefficients partiels $\gamma_{\mathrm{F}}$ sur les actions pour la combinaison type fondamentale (en situations de projet durables ou transitoires).

Pour les situations de projet accidentelles, on ajoute la valeur nominale de l'action accidentelle aux charges permanentes et variables non pondérées, c'est-à-dire que tous les $\gamma_{\mathrm{F}}=1,0$.

L'EN 1997-1 (CEN, 2004) propose trois approches (1, 2 et 3 ) : pour l'approche 1 , il y a lieu d'effectuer deux

\begin{tabular}{|c|c|c|c|c|c|c|}
\hline \multicolumn{2}{|c|}{ Actions } & \multicolumn{2}{|c|}{$\begin{array}{l}\text { EurOCODE } \\
\text { Approche } 1 \\
\text { Calcul 1 (B) Calcul } 2(\mathrm{C})^{* *}\end{array}$} & $\begin{array}{l}\text { valeurs recor } \\
\text { Approche } 2\end{array}$ & $\begin{array}{l}\text { dées) } \\
\text { Approche } 3^{*}\end{array}$ & FASCICULE $62-\mathrm{V}^{* * * *}$ \\
\hline Permanentes & $\begin{array}{l}\text { Défavorables } \\
\text { Favorables }\end{array}$ & $\begin{array}{l}1,35 \\
1,0\end{array}$ & $\begin{array}{l}1,0 \\
1,0\end{array}$ & $\begin{array}{c}1,35 \\
1,0\end{array}$ & $\begin{array}{c}1,35 \\
1,0\end{array}$ & $\begin{array}{c}1,35 \\
1,0\end{array}$ \\
\hline Variables & Défavorables & 1,5 & 1,3 & 1,5 & 1,5 & 1,5 \\
\hline
\end{tabular}
calculs et retenir le résultat le plus défavorable (anciens

TABLEAUIV Coefficients partiels $\gamma_{F}$. Combinaison fondamentale (ELU, situations de projet durables ou transitoires). Partial factors $\gamma_{\mathrm{F}}$. Fundamental combination (ULS, persistent or transient design situations).

* Pour l'approche 3 : coefficients à appliquer aux actions provenant des structures portées uniquement, avec pondération « à la source » des propriétés des sols intervenant dans le calcul des actions.

** Pondération « à la source » des propriétés des sols intervenant dans le calcul des actions.

*** Fascicule $62-\mathrm{V}$ : ne traite pas des ouvrages de soutènement, ni donc des actions qui s'y exercent.

Note: Pour l'Eurocode 7, la pondération peut être appliquée aux actions ou directement à l'effet des actions. 
cas B et C de la pré-norme pour l'Eurocode 7-1: ENV 1997-1, 1994, voir AFNOR, 1996).

L'approche du Fascicule 62-V est identique à l'approche 2 de l'EN 1997-1.

Les coefficients partiels $\gamma_{5}$ du Fascicule 62 - V sont identiques à ceux de l'EN 1997, à l'exception d'une part du calcul 2 de l'approche 1, et d'autre part en ce qui concerne les charges d'exploitation étroitement bornées ou de caractère particulier pour lesquelles $\gamma_{\mathrm{F}}=1,35$ au lieu de 1,50.

Le tableau $\mathrm{V}$ donne les coefficients partiels appliqués à la résistance du pieu, c'est-à-dire les valeurs de $\gamma$ (ou $\gamma_{b}$ et $\gamma_{s}$ ) pour la combinaison type fondamentale.

Dans le Fascicule 62-V on utilise les mêmes valeurs $\gamma_{\mathrm{b}}$ et $\gamma_{\mathrm{s}}$ pour la résistance de pointe et pour la résistance de fût, quel que soit le type de pieu: pour la combinaison fondamentale, cette valeur est $\gamma_{\mathrm{s}}=\gamma_{\mathrm{b}}=\gamma_{\mathrm{t}}=1,40$.

La combinaison type d'actions pour les situations de projet accidentelles est la même dans l'EN 1997-1 et le Fascicule 62-V, avec $\gamma_{\mathrm{F}}=1,0$ (pour plus de détails, on pourra se reporter par exemple à Frank, 1994).

Pour les situations de projet accidentelles, l'EN 1997-1, ne recommande pas de valeurs pour les coefficients $\gamma_{t}, \gamma_{b}$ et $\gamma_{s}$. Par la suite, pour l'application de l'EN 1997-1, on supposera (en cohérence avec l'EN 1990 Eurocode: Bases du calcul des structures, CEN, 2003) qu'ils sont égaux à 1,0. Dans le Fascicule 62-V le coefficient $\gamma$, est égal à 1,20.

\section{3}

\section{Diverses natures de coefficients de sécurité}

\section{1}

\section{Coefficients " globaux » de sécurité}

On peut écrire, en résumé, avec une notation synthétique issue des précédentes formulations, dans le cas des essais de chargement statique (§ 2.2.1) et dans celui des essais de sol - méthode “ directe $)(§ 2.2 .2 .2)$, selon l'EN 1997-1 et le Fascicule 62-V:

$\mathrm{R}_{\mathrm{c}, \mathrm{k}}=\mathrm{R}_{\mathrm{c} \text {, mes }} / \xi$ (essais de chargement statique) (7.1)

$\mathrm{R}_{\mathrm{c}, \mathrm{k}}=\mathrm{R}_{\mathrm{c}, \mathrm{cal}} / \xi$ (essais de sol - méthode « directe »)(7.2) (dans ce dernier cas de figure, $\xi$ n'est pas présenté de façon explicite).

La condition d'état limite de défaut de capacité portante s'exprime de façon « canonique ») par:

$$
\mathrm{F}_{\mathrm{c}, \mathrm{d}} \leq \mathrm{R}_{\mathrm{c}, \mathrm{d}}
$$

Les relations (5) à (8) conduisent alors à réexprimer la condition d'état limite en faisant apparaître un coefficient de sécurité global:

$$
\begin{gathered}
\mathrm{F}_{\mathrm{c}, \mathrm{k}} \leq\left(\mathrm{R}_{\mathrm{c}, \text { mes }} / \xi\right) /\left(\gamma_{\mathrm{F}} \cdot \gamma_{\mathrm{t}}\right)=\mathrm{R}_{\mathrm{c}, \text { mes }} / \mathrm{FS} \\
\mathrm{F}_{\mathrm{c}, \mathrm{k}} \leq\left(\mathrm{R}_{\mathrm{c}, \mathrm{cal}} / \xi\right) /\left(\gamma_{\mathrm{F}} \cdot \gamma_{\mathrm{t}}\right)=\left(\mathrm{R}_{\mathrm{c}, \mathrm{cal}} / \xi\right) / \mathrm{FS}^{\prime}
\end{gathered}
$$

avec la notation synthétique $\gamma_{\mathrm{F}}$ pour les coefficients partiels sur les actions et $\gamma_{\mathrm{t}}$ pour les coefficients partiels sur la résistance (remplaçant éventuellement $\gamma_{b}$ et $\gamma_{s}$ ).

Nous notons FS $=\gamma_{\mathrm{F}} \cdot \gamma_{\mathrm{r}}, \boldsymbol{\xi}$ le coefficient de sécurité global par rapport à la valeur des résistances mesurées dans des essais de chargement statique de pieux (relation 9.1).

Dans le cas de l'utilisation des résultats d'essais de sol, en l'absence de dispositions comparables dans le Fascicule $62-V$, nous ne définissons pas de coefficient global de sécurité pour la méthode des « pieux modèle ») du $§ 2.2 .2 .1$.

Nous préférons définir FS' $=\gamma_{\mathrm{F}} \cdot \gamma_{\mathrm{t}}$ (relation 9.2) le coefficient de sécurité global par rapport à la valeur caractéristique de la résistance axiale du pieu (résistance de pointe et frottement latéral) telle qu'elle est évaluée directement par le modèle de prévision (cas du Fascicule 62-V et cas de la méthode (c alternative ») de l'EN 1997-1) c'est-à-dire à partir des valeurs des propriétés des sols (voir $\$ 2.2 .2 .3$ ). Dans ce cas les valeurs de $\xi$ ne sont pas explicitées car elles sont censées être incluses dans $\mathrm{q}_{\mathrm{b}, \mathrm{k}}$ et $\mathrm{q}_{\mathrm{s}, \mathrm{k}}$.

\section{2}

\section{Coefficients de modèle}

Dans la théorie du semi-probabilisme, les $\gamma_{F}$ et $\gamma_{R}$ sont en fait le résultat du produit de deux coefficients partiels: le coefficient ( de valeur ») et le coefficient « de modèle ». Pour mettre en évidence ce dernier, noté $\gamma_{S d}$ pour le modèle d'actions et $\gamma_{\mathrm{Rd}}$ pour le modèle de résistance, l'on réécrit les inégalités (9.1) et (9.2) en faisant apparaître l'expression développée des coefficients partiels du côté des actions et des résistances (notations des Eurocodes):

$$
\begin{aligned}
& \gamma_{\mathrm{F}}=\gamma_{\mathrm{Sd}} \cdot \gamma_{\mathrm{f}} \\
& \gamma_{\mathrm{R}}=\gamma_{\mathrm{Rd}} \cdot \gamma_{\mathrm{r}}
\end{aligned}
$$

Les coefficients $\gamma_{\mathrm{F}}$ usuels de 1,35 et 1,50 se décomposent en des coefficients $\gamma_{\text {f }}$ (coefficients de valeur) de 1,20 et 1,33, multipliés par le coefficient de modèle d'action sous-jacent $\gamma_{\mathrm{Sd}}$ de 1,125 analogue au $\gamma_{\mathrm{F} 3}$ des règlements français de béton et de fondations (Mathieu, 1975). Nous utilisons dans la suite cette valeur de $\gamma_{\mathrm{Sd}}=1,125$ qui est transposable telle quelle au format des Eurocodes (approche 2) car, comme nous l'avons vu précédemment, les deux règlements ne diffèrent pas sur les coefficients à considérer du côté des

\begin{tabular}{|c|c|c|c|c|c|c|c|c|c|}
\hline \multirow{3}{*}{ Type de pieu } & \multirow{2}{*}{\multicolumn{3}{|c|}{ Calcul 1 (B) }} & \multicolumn{4}{|c|}{ EUROCODE 7 (valeurs recommandées) } & \multirow[b]{2}{*}{ Approche $3^{*}$} & \multirow[b]{2}{*}{ FASCICULE 62-V } \\
\hline & & & & & lcul: & & Approche 2 & & \\
\hline & $\gamma_{\mathrm{b}}$ & $\gamma_{\mathrm{s}}$ & $\gamma_{t}$ & $\gamma_{b}$ & $\gamma_{\mathrm{s}}$ & $\gamma_{\mathrm{t}}$ & $\gamma_{\mathrm{b}}=\gamma_{\mathrm{s}}=\gamma_{\mathrm{t}}$ & $\gamma_{\mathrm{b}}=\gamma_{\mathrm{s}}=\gamma_{\mathrm{t}}$ & $\gamma_{\mathrm{b}}=\gamma_{\mathrm{s}}=\gamma_{\mathrm{t}}$ \\
\hline Pieu battu & 1,0 & 1,0 & 1,0 & 1,3 & 1,3 & 1,3 & 1,1 & 1,0 & 1,4 \\
\hline Pieu foré & 1,25 & 1,0 & 1,15 & 1,6 & 1,3 & 1,5 & 1,1 & 1,0 & 1,4 \\
\hline Tarière continue & 1,1 & 1,0 & 1,1 & 1,45 & 1,3 & 1,4 & 1,1 & 1,0 & 1,4 \\
\hline
\end{tabular}
actions. Les valeurs de $\gamma_{\mathrm{F}}$ du tableau IV regroupent

TABLEAUV Coefficients partiels $\gamma_{\mathrm{b}}, \gamma_{\mathrm{s}}$ et $\gamma_{\mathrm{t}}$ pour les pieux en compression. Combinaison fondamentale (ELU, situations de projet durables ou transitoires).

Partial factors $\gamma_{b}, \gamma_{s}$ and $\gamma_{t}$ for piles in compression (ULS, persistent or transient design situations).

* L'approche 3 n'est pas applicable à la méthode de calcul de la résistance basée sur les essais de pieux. 
dans une formulation condensée les incertitudes de valeur et les incertitudes de modèle ainsi définies.

On définit le coefficient ( réuni ») de modèle par le produit $\gamma_{\mathrm{d}}=\gamma_{\mathrm{Sd}} \cdot \gamma_{\mathrm{Rd}}$. Le coefficient réuni de modèle est présenté par Kovarik (2000).

Dans l'interprétation que les auteurs font de l'EN 1997-1 et du Fascicule 62-V, les coefficients partiels $\gamma_{t} \gamma_{\mathrm{b}}$ et $\gamma_{\mathrm{s}}$ introduits au $\S 2.3$ s'assimilent, comme on l'a vu, à des coefficients de type ( $\left.\gamma_{\mathrm{B}}\right)$, mais avec un coefficient de modèle de résistance sous-jacent $\gamma_{\mathrm{Rd}}$ de 1,00. Comme le propos de cet article est de caler les coefficients $\gamma_{\mathrm{Rd}}$ donc de leur donner des valeurs qui auront toutes les chances d'être différentes de 1,00 , nous convenons expressément de considérer dans la suite les $\gamma_{t^{\prime}} \gamma_{\mathrm{b}}$ et $\gamma_{\mathrm{s}}$ comme des ( $\left.\gamma_{r}\right)$ ) (c'est-à-dire comme des coefficients de valeur) et par simplicité nous continuerons à les noter de façon générale par « $\left.\gamma_{t}\right)$.

D'où des expressions plus développées des conditions d'état limite (9.1) et (9.2) et des coefficients globaux de sécurité:

$$
\begin{aligned}
& \mathrm{F}_{c, k} \leq \mathrm{R}_{\mathrm{c} \text {, mes }} /\left(\gamma_{\mathrm{Sd}} \cdot \gamma_{\mathrm{Rd}} \gamma_{\mathrm{f}} \cdot \gamma_{\mathrm{t}} \cdot \xi\right) \\
& \mathrm{F}_{\mathrm{c}, \mathrm{k}} \leq\left(\mathrm{R}_{\mathrm{c}, \text { cal }} / \xi\right) /\left(\gamma_{\mathrm{Sd}} \cdot \gamma_{\mathrm{Rd}} \gamma_{\mathrm{f}} \cdot \gamma_{\mathrm{t}}\right)
\end{aligned}
$$

Les coefficients globaux de sécurité deviennent FS = $\gamma_{\mathrm{Sd}} \gamma_{\mathrm{Rd}} \gamma_{\mathrm{f}} \gamma_{\mathrm{t}}, \xi$ pour des essais de chargement statique de pieux, et $\mathrm{FS}^{\prime}=\gamma_{\mathrm{Sd}} \gamma_{\mathrm{Rd}} \gamma_{\mathrm{f}} \gamma_{\mathrm{t}}$ dans la méthode de prévision alternative ( directe » à partir des propriétés des sols.

Pour formelle que paraisse à première vue cette décomposition des coefficients partiels, l'avantage pratique pour le rédacteur de normes et règlements est d'abord de permettre un calage adapté de chaque coefficient partiel, de valeur et de modèle, en application du principe du « traitement des incertitudes là où elles se trouvent ).

\section{4 \\ Exemples de comparaison des niveaux globaux de sécurité}

Dans ce qui suit, on compare les valeurs du coefficient global de sécurité FS ou FS' donné au § 3.1, dans le cas de l'EN 1997-1 (noté FS $\mathrm{EC7}_{\mathrm{EC}}$ ou $\mathrm{FS}_{\mathrm{EC7}}^{\prime}$ ) et du Fascicule $62-\mathrm{V}$ (noté $\mathrm{FS}_{62 \mathrm{~V}}$ ou $\mathrm{FS}_{62 \mathrm{~V}}^{\prime}$ ), pour les situations de projet durables et transitoires d'une part, pour les situations de projet accidentelles d'autre part.

Il n'est pas tenu compte de l'approche 3 de l'EN 1997-1 qui utilise la pondération à la source des paramètres élémentaires de la résistance des sols pour déterminer le frottement latéral et la résistance de pointe, car le Fascicule-V se place dans la cas de figure où la résistance du pieu est déterminée, par essais de pieux ou essais in situ des sols, sans passer par les propriétés élémentaires des sols.

Les résultats des calculs sont repris dans les tableaux synthétiques du $§ 5.2$.

\section{1}

\section{Calcul sur la base d'essais de chargement statique de pieux}

Après un cas simple, on étudie deux exemples qui proviennent des études de dimensionnement des fonda- tions du pont Vasco de Gama (sur le Tage, à Lisbonne). L'ouvrage comporte, en fait, plusieurs ponts et viaducs importants fondés sur pieux (Wastiaux et al., 1998 et 1999). Le calcul de pieux effectué à l'époque a suivi les prescriptions de la pré-norme ENV 1997-1 (1994, voir AFNOR, 1996). La présentation qui en est faite ci-dessous tient compte des évolutions de l'EN 1997-1 (CEN, 2004).

Les valeurs numériques des tableaux I et II sont utilisées pour $\xi$ tant pour les situations de projet durables et transitoires (combinaisons fondamentales) que pour les situations de projet accidentelles. Les valeurs numériques des tableaux IV et V sont utilisées en situations de projet durables et transitoires. Pour les situations de projet accidentelles, on utilise $\gamma_{F}=1,0$ dans tous les cas et $\gamma_{\mathrm{t}}=1,0$ pour l'EN 1997-1 et $\gamma_{\mathrm{t}}=1,2$ pour le Fascicule $62-\mathrm{V}$.

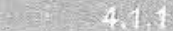 \\ Un cas (théorique) simple}

On considère un pieu exécuté à la tarière continue et on suppose que les résultats d'un seul essai de chargement statique sont disponibles, sans mesures séparées des résistances de pointe et de fût (on utilise donc, tant pour l'EN 1997-1 que pour le Fascicule 62-V, le coefficient $\gamma_{t}$ ). Par ailleurs, les charges sont supposées être à $100 \%$ des charges permanentes défavorables, auxquelles se rajoute une action accidentelle quelconque (non pondérée) dans la combinaison accidentelle.

- Combinaison fondamentale:

EN 1997-1:

Approche 1

$$
\begin{aligned}
& \text { Calcul 1 (B): } \mathrm{FS}_{\mathrm{EC7}}=1,35 \times 1,10 \times 1,40=2,08 \\
& \text { Calcul 2 (C): } \mathrm{FS}_{\mathrm{EC7}}=1,00 \times 1,40 \times 1,40=1,96
\end{aligned}
$$

C'est le calcul 1(B) qui est le plus défavorable et qu'il $\mathrm{y}$ a donc lieu de retenir.

Approche $2 \quad \mathrm{FS}_{\mathrm{EC} 7}=1,35 \times 1,10 \times 1,40=2,08$

Fascicule $n^{\circ} 62$ - Titre V:

$$
\mathrm{FS}_{62 \mathrm{~V}}=1,35 \times 1,40 \times 1,20=2,27
$$

- Combinaison accidentelle:

EN 1997-1:

$$
\mathrm{FS}_{\mathrm{EC} 7}=1,00 \times 1,00 \times 1,40=1,40
$$

Fascicule $\mathrm{n}^{\circ} 62$ - Titre $\mathrm{V}$ :

$$
\mathrm{FS}_{62 \mathrm{~V}}=1,00 \times 1,20 \times 1,20=1,44
$$

La sécurité des deux règlements est très voisine, le Fascicule $62-\mathrm{V}$ étant légèrement plus sécuritaire pour la combinaison fondamentale $(+9 \%)$.

\section{9 \\ Pieux forés, résultats d'un seul essai de chargement statique}

- Combinaison fondamentale et $50 \%$ de charges permanentes et $50 \%$ de charges variables défavorables. Pour simplifier, on applique dans tous les cas le coefficient $\gamma_{\text {s. }}$

\section{EN 1997-1:}

\section{Approche 1}

$$
\text { Calcul } 1 \text { (B): } \text { FS }_{E C 7}=1,425 \times 1,15 \times 1,40=2,29
$$$$
\text { Calcul } 2(\mathrm{C}): \mathrm{FS}_{\mathrm{EC7}}=1,150 \times 1,50 \times 1,40=2,42
$$

C'est le calcul 2(C) qui est le plus défavorable et qu'il $\mathrm{y}$ a donc lieu de retenir. 


\section{Approche 2}

$$
\mathrm{FS}_{\mathrm{EC7}}=1,425 \times 1,10 \times 1,40=2,19
$$

Fascicule $\mathrm{n}^{\circ} 62-$ Titre $\mathrm{V}$ :

$$
\mathrm{FS}_{62 \mathrm{~V}}=1,425 \times 1,40 \times 1,20=2,39
$$

- Combinaison accidentelle :

EN 1997-1:

$$
\mathrm{FS}_{\mathrm{EC7}}=1,00 \times 1,00 \times 1,40=1,40
$$

Fascicule $n^{\circ} 62$ - Titre $V$ :

$$
\mathrm{FS}_{62 \mathrm{~V}}=1,00 \times 1,20 \times 1,20=1,44
$$

Ici également, la sécurité des deux règlements est très voisine, le Fascicule 62-V étant légèrement plus sécuritaire que l'approche 2 de l'EN 1997-1 (pour la combinaison fondamentale, $+9 \%$ ).

\section{Pieux battus, résultats de 4 essais de chargement statique}

On suppose que c'est la valeur moyenne des résistances mesurées qui gouverne et, dans le cas de l'EN 1997-1, on applique donc $\xi_{1}=1,10$ (Tableau I). Dans le cas du Fascicule 62-V, la relation (3) et les valeurs $R_{c}$ et $R_{c, \max }$ du projet reviennent à appliquer $\xi=1,14$ sur le valeur moyenne des résistances mesurées.

- Combinaison fondamentale et $67 \%$ de charges permanentes et $33 \%$ de charges variables défavorables:

EN 1997-1:

Approche 1

$$
\begin{aligned}
& \text { Calcul } 1(\mathrm{~B}): \mathrm{FS}_{\mathrm{EC7}}=1,40 \times 1,00 \times 1,10=1,54 \\
& \text { Calcul } 2(\mathrm{C}): \mathrm{FS}_{\mathrm{EC7}}=1,10 \times 1,30 \times 1,10=1,57
\end{aligned}
$$

C'est le calcul 2(C) qui est le plus défavorable et qu'il y a donc lieu de retenir.

Approche 2

$$
\mathrm{FS}_{\mathrm{EC} 7}=1,40 \times 1,10 \times 1,10=1,69
$$

Notons qu'en utilisant l'EN 1997-1 on est assuré d'avoir au moins $\mathrm{FS}=1,43$ par rapport à la résistance la plus basse mesurée $\left(\xi_{2}=1,00\right)$.

Fascicule $\mathrm{n}^{\circ} 62$ - Titre $\mathrm{V}$ :

$$
\mathrm{FS}_{62 \mathrm{~V}}=1,40 \times 1,40 \times 1,14=2,23
$$

La sécurité préconisée par le Fascicule 62-V est nettement plus importante que dans le cas de l'EN 1997-1: + $32 \%$ par rapport à l'approche 2 . La différence de niveau de sécurité ne provient pas tant des valeurs de $\xi$ que de celles des coefficients partiels de valeur $\gamma_{F}$ et $\gamma_{t}$. - Combinaison accidentelle:

EN 1997-1:

$$
\mathrm{FS}_{\mathrm{EC} 7}=1,00 \times 1,00 \times 1,10=1,10
$$

(au moins $\mathrm{FS}=1,00$ par rapport à la résistance la plus basse mesurée).

Fascicule $n^{\circ} 62$ - Titre V:

$$
\mathrm{FS}_{62 \mathrm{~V}}=1,00 \times 1,20 \times 1,14=1,37
$$

La sécurité du Fascicule 62-V est plus importante que dans le cas de l'EN 1997-1: + 25\% pour des raisons analogues à celles expliquées plus haut dans le cas de la combinaison fondamentale.

\section{2}

\section{Calcul sur la base d'essais de sol}

Les règles pressiométriques ou pénétrométriques actuelles du Fascicule 62-V (MELT, 1993) sont supposé être utilisées dans tous les cas. On détermine le FS': c'est-à-dire que l'on ne considère pas les coefficients $\xi$ de l'Eurocode 7 qui ne servent qu'à déterminer une valeur caractéristique dans la méthode des « pieux modèle 》, cette dernière ne pouvant être examinée ici faute de disposer pour elle d'élément de comparaison avec le Fascicule 62-V.

Les valeurs numériques des tableaux IV et $\mathrm{V}$ sont utilisées en situations de projet durables et transitoires. Pour les situations de projet accidentelles, on utilise $\gamma_{\mathrm{F}}=1,0$ dans tous les cas et $\gamma_{\mathrm{f}}=1,0$ pour I'EN 1997-1 et $\gamma_{\mathrm{t}}=1,2$ pour le Fascicule 62-V.

\section{- Combinaison fondamentale}

Toutes les actions sont considérées comme défavorables. Pour l'EN 1997-1, on utilise les mêmes coefficients $\gamma$ pour les résistances de pointe et de fût $\left(\gamma_{b}=\gamma_{s}=\gamma_{t}\right)$, comme c'est le cas dans le Fascicule 62-V.

\section{EN 1997-1:}

On détaille ci-dessous le calcul le plus défavorable, c'est-à-dire celui qui donne $\mathrm{FS}^{\prime}$ le plus grand: dans le cas de charges permanentes seules, c'est le calcul 1 (B) et dans le cas où une certaine proportion de charges variables serait appliquée, c'est le calcul 2 (C).

\section{Approche 1}

Calcul 1 (B) :

- pieu battu, $100 \%$ de charges permanentes: $\mathrm{FS}_{\mathrm{EC} 7}^{\prime}=1,35 \times 1,00=1,35$

- pieu foré, $100 \%$ de charges permanentes:

Calcul 2 (C) :

$$
\mathrm{FS}_{\mathrm{EC} 7}^{\prime}=1,35 \times 1,15=1,55
$$

- pieu battu, $50 \%$ de charges permanentes et $50 \%$ de charges variables:

$$
\mathrm{FS}^{\prime}{ }_{\mathrm{EC} 7}=1,15 \times 1,30=1,50
$$

- pieu foré, $50 \%$ de charges permanentes et $50 \%$ de charges variables :

$$
\mathrm{FS}_{\mathrm{EC} 7}^{\prime}=1,15 \times 1,50=1,725
$$

Approche 2

- pieux battu et foré:

$100 \%$ de charges permanentes:

$$
\mathrm{FS}^{\prime}{ }_{\mathrm{EC} 7}=1,35 \times 1,10=1,485
$$

$50 \%$ de charges permanentes et $50 \%$ de charges variables:

$$
\mathrm{FS}_{\mathrm{EC7}}^{\prime}=1,425 \times 1,10=1,5
$$

Fascicule $n^{\circ} 62$ - Titre V:

$100 \%$ de charges permanentes :

$$
\mathrm{FS}^{\prime}{ }_{62 \mathrm{~V}}=1,35 \times 1,40=1,89
$$

$50 \%$ de charges permanentes et $50 \%$ de charges variables:

$$
\mathrm{FS}_{62 \mathrm{~V}}^{\prime}=1,425 \times 1,40=1,995
$$

- Combinaison accidentelle

Tous les $\gamma_{\mathrm{F}}=1,00$.

EN 1997-1:

$$
\mathrm{FS}_{\mathrm{EC7}}^{\prime}=1,00 \times 1,00=1,00
$$

Fascicule $n^{\circ} 62$ - Titre $V$ :

$$
\mathrm{FS}_{62 \mathrm{~V}}^{\prime}=1,00 \times 1,20=1,20
$$

\section{Commentaires}

On constate que le Fascicule $62-\mathrm{V}$ se révèle globalement plus sécuritaire que l’EN 1997-1. 
En fait l'EN 1997-1 introduit une certaine discontinuité avec la pratique courante. Le propos va être, dans la suite de cet article, d'introduire un " coefficient de modèle » facilitant les raccordements, de lui donner un sens et de lui attribuer une valeur numérique.

\section{5}

\section{Calages du coefficient de modèle de résistance (approche 2)}

\section{1}

\section{Position du problème}

Comment déterminer la valeur du coefficient de modèle $\gamma_{R d}$ (relation 10.2) qui serait appliqué dans le format de l'EN 1997-1 avec les $\gamma$, qu'il prévoit (rappelons que notre $\gamma_{\mathrm{r}}$ est l'équivalent de la notation $\gamma_{\mathrm{r}}$ des Eurocodes, voir \$ 3.2 ) et qui conduirait à un « niveau de sécurité ») analogue à celui du Fascicule 62-V? Nous noterons cette valeur " $\gamma_{\text {Rd, calé }}$ ).

Le calage par ajustement, une fois donnés tous les autres coefficients partiels de valeur, revient à déterminer le $\gamma_{\text {Rd, calé }}$ pour que le niveau global de sécurité de la fondation soit aussi proche que possible du niveau de sécurité de référence choisi, par exemple celui du Fascicule 62-V dans le cadre d'un ajustement national (§ 5.2).

Par ailleurs, comment traduire dans la valeur du coefficient partiel de résistance $\gamma_{R}$ (relation 5.0) l'information obtenue par une série de comparaisons effectuées entre les prévisions du modèle de résistance (modèle pressiométrique, par exemple) et la réalité (mesurée) de la capacité portante de la fondation (résultats des essais de chargement statique de » pieux)? Nous noterons cette valeur " $\gamma_{\mathrm{R} \text {, calé }}$ ).

Une fois donnés tous les autres coefficients partiels de valeur, le calage probabiliste consiste à déterminer le $\gamma_{\mathrm{R} \text {, cale }}$ pour l'état limite de défaut de capacité portante, de telle sorte que la fiabilité de la fondation ainsi dimensionnée soit aussi proche que possible d'une fiabilité cible donnée par référence à une probabilité de ruine (§ 5.3).

\section{2}

\section{Calage par ajustement sur la base du niveau de sécurité actuel du Fascicule 62-V}

Ce calage est applicable au cas où l'on utilise les résultats d'essais de sol et au cas où l'on utilise des résultats d'essais de pieux.

\section{1}

\section{Coefficient de modèle de résistance}

Dans le cadre de la détermination nationale des paramètres des Eurocodes touchant les niveaux de sécurité des ouvrages, on indique dans cette partie comment procéder au calage par ajustement du coefficient de modèle: ce coefficient $\gamma_{\text {Bd alé }}$ utilisé avec les autres coefficients partiels de l'EN 1997-1 (approche 2) permettra de retrouver les niveaux de sécurité nationaux du Fascicule 62-V.

Avec les expressions développées au paragraphe 3.2, le niveau de sécurité actuel du Fascicule 62-V s'exprime par " $\gamma_{\mathrm{Rd}}$. $\mathrm{FS}_{62 \mathrm{~V}}$ ) (avec $\gamma_{\mathrm{Rd}}=1,00$ comme exposé ci-dessus).

Le niveau de sécurité de l'EN 1997-1 s'exprimera par ( $\gamma_{\mathrm{Rd}} \cdot \mathrm{FS}_{\mathrm{EC} 7}$ ) (avec $\gamma_{\mathrm{Rd}}$ à caler).

Le coefficient de modèle du côté des résistances, à employer avec le format de l'EN 1997-1 pour conduire à l'ajustement des niveaux de sécurité, est donc déterminé par:

$$
\gamma_{\mathrm{Rd}, \text { calé }}=\mathrm{FS}_{62 \mathrm{~V}} / \mathrm{FS}_{\mathrm{EC7}}
$$

L'exploitation des résultats numériques de la partie précédente donne accès à une fourchette de valeurs pour ce coefficient de modèle (Tableau VIa). Rappelons que la comparaison est menée: dans le cas basé sur les essais de pieux, par référence à la valeur moyenne des essais; dans le cas basé sur les essais de sol, par référence à la valeur caractéristique de la résistance axiale du pieu.

Le début d'étude de sensibilité qui a été engagé dans ce cadre conduit, on le voit, à différents coefficients $\gamma_{\mathrm{Rd} \text {, calé }}$ pour la méthode des essais de pieux: dans le cas d'un calage par ajustement, le coefficient de modèle dépend du nombre d'essais de pieux (à l'instar du coefficient de résistance) : c'est une incohérence par rapport au format semiprobabiliste considéré avec rigueur. Cette incohérence disparaîtrait si l'on effectuait un calage du coefficient de modèle par référence au FS' au lieu du FS, c'est-à-dire si le coefficient de réduction statistique $\xi$ était intégré à la valeur caractéristique de la résistance (il intervient aussi bien dans les combinaisons fondamentales et accidentelles que dans celles qui sont associées au états limites de service).

\section{Coefficient réuni de modèle}

On en déduit le coefficient réuni de modèle (calé) qui, dans les situations de projet durables et transitoires d'une part, dans les situations de projet accidentelles d'autre part, conduit à l'ajustement des niveaux de sécurité des deux (c codes ) de calcul (Tableau VIb):

$$
\begin{aligned}
& \gamma_{\mathrm{d} \text {, calé, fond }}=\gamma_{\mathrm{Sd} \text {, fond }} \cdot \gamma_{\mathrm{Rd} \text {, calé, fond }}=1,125 \cdot\left[\mathrm{FS}_{62 \mathrm{~V}} / \mathrm{FS}_{\mathrm{EC7}}\right]_{\mathrm{fond}} \\
& \gamma_{\mathrm{d} \text {, calé, acc }}=\gamma_{\mathrm{Sd} \text {, acc }} \cdot \gamma_{\mathrm{Rd} \text {, calé, acc }}=1,00 \cdot\left[\mathrm{FS}_{62 \mathrm{~V}} / \mathrm{FS}_{\mathrm{EC7}}\right]_{\mathrm{acc}}
\end{aligned}
$$

TABLEAU VIa Coefficient de modèle $\gamma_{\text {Rd, calé }}$ pour ajuster le niveau de sécurité de l'EN 1997-1 à celui du Fascicule 62-V. Model factor $\gamma_{\mathrm{Bd}}$, calib to adjust the safety model of EN 1997-1 to the one of Fascicule 62-V.

A partir d'essais de pieux (FS)

Cas théorique simple Pieu foré, 1 essai
A partir d'essais de sol (FS')

sous la combinaison fondamentale

$\mathrm{FS}_{\mathrm{EC} 7}$ (appr. 2)

$\mathrm{FS}_{62 \mathrm{~V}}$

$\gamma_{\mathrm{Rd}, \text { cale, fond }}$

FS $_{\text {EC7 }}$ (appr. 2)

$\mathrm{FS}_{62 \mathrm{~V}}$

$\gamma_{\mathrm{Rd} \text {, calé, acc }}$
2,08

2,27

1,093

1,40

1,44

1,03

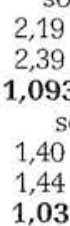

2,19
2,39

1,093 so

1,44

1,03
1,69

2,23

1,316

1,10

1,37

1,25
$1,1 \gamma_{\mathrm{F}}$

$1,4 \gamma_{\mathrm{F}}$

1,273

1,00

1,20

1,20 
TABLEAU VIb Coefficient réuni de modèle $\gamma_{\text {d, calé }}$ pour ajuster le niveau de sécurité de l'EN 1997-1 à celui du Fascicule 62-V. Unified model factor $\gamma_{d}$, calib to adjust the safety model of EN 1997-1 to the one of Fascicule 62-V.

\section{A partir d'essais de pieux (FS)}

Cas théorique simple Pieu foré, 1 essai Pieu battu, 4 essais

A partir d'essais de sol (FS')

\begin{tabular}{|c|c|c|c|c|}
\hline \multirow[b]{2}{*}{$\gamma_{\text {Bd als fond }}$} & \multicolumn{4}{|c|}{ sous la combinaison accidentelle } \\
\hline & 1,093 & 1,093 & 1,316 & 1,273 \\
\hline $\mathrm{V}_{\mathrm{Sd} \text {, fond }}$ & 1,125 & 1,125 & 1,125 & 1,125 \\
\hline$\gamma_{d, \text { calé, fond }}$ & 1,23 & 1,23 & 1,48 & 1,43 \\
\hline & 1,03 & $\begin{array}{r}\text { so } \\
1.03\end{array}$ & $\begin{array}{c}\text { cident } \\
1.25\end{array}$ & 120 \\
\hline $\begin{array}{l}I_{\text {Rd, calé, acc }} \\
\gamma_{\mathrm{Sd}, \text { acc }}\end{array}$ & 1,00 & 1,00 & 1,00 & 1,00 \\
\hline Yate & 1,03 & 1,03 & 1,25 & 1,20 \\
\hline
\end{tabular}

\section{3}

\section{Calage probabiliste prenant en compte une évaluation statistique de l'erreur de modèle et la variabilité spatiale de la résistance}

L'incertitude concernant la résistance d'un pieu provient de trois sources principales:

- l'incertitude du modèle de calcul, c'est-à-dire l'incertitude propre aux facteurs et abaques de calcul reliant la pression limite pressiométrique à la résistance de pointe et de fût;

- l'incertitude concernant la variabilité des propriétés pressiométriques, liée à la variabilité des couches et des propriétés des sols en place;

- l'incertitude d'échantillonnage due à ce que les estimations des paramètres et lois de probabilité sont faits à partir d'un nombre forcément limité de points de reconnaissance ou d'essais.

Ces trois sources d'incertitude sont indépendantes et doivent être composées pour décrire l'incertitude globale de la résistance. Dans ce qui suit, on décrit les deux premières incertitudes respectivement au moyen d'une distribution $\hat{\eta}$ (incertitude due au modèle de calcul) et d'une distribution $\hat{\mathrm{p}}$ (incertitude due à la variabilité spatiale), l'incertitude d'échantillonnage étant considérée comme déjà intégrée à l'estimation de leurs paramètres.

Dans ce calage, on révise le niveau de sécurité actuel du Fascicule 62-V et de l'EN 1997-1. Ce type de calage qui globalise les sources d'incertitude tant en ce qui concerne la valeur caractéristique que la valeur de calcul, est transposable à tout modèle à partir d'essais de sol, mais pour la méthode « directe») (ou « alternative ») seulement.

La méthode des « pieux-modèle » telle qu'elle a été exposée plus haut, traite quant à elle ces trois sources d'incertitude par deux coefficients distincts : le coefficient $\xi$ considère déjà la variabilité spatiale et l'incertitude d'échantillonnage (il sert à la détermination de la valeur caractéristique), le coefficient de modèle - à introduire - considérerait exclusivement l'incertitude de modèle.

\section{Modélisation de l'erreur de modèle}

La réalité de la résistance d'un pieu dépend de nombreux facteurs dont un nombre limité seulement est considéré dans un modèle de calcul. Les facteurs non pris en compte font apparaître la réalité comme « brui- tée », ce que l'on modélise par une variable aléatoire, la réalisation de ce bruit étant incertaine.

Le coefficient $\gamma_{R d}$ d'un modèle de résistance donné (à partir de résultats d'essais de pieux, d'essais de sol) est choisi de manière à couvrir cette imprécision du modèle (autrement dit les facteurs non pris en compte dans le modèle). Les incertitudes sur l'exécution des pieux, sur les propriétés de résistance au droit des futurs pieux (autrement dit l'incertitude sur les facteurs explicitement pris en compte dans le modèle) sont couvertes quant à elles par les coefficients de sécurité ( de valeur ».

On note $m$ et s la moyenne et l'écart type empirique d'un ensemble de valeurs; on note $\mu$ et $\sigma$ la moyenne et l'écart type d'une loi de distribution.

Lorsque l'on dispose à la fois de résultats d'essais de résistance à la compression, $\mathrm{R}_{\mathrm{c}, \text { mes' }^{\prime}}$ mesurée par des essais de chargement statique et de valeurs calculées de la résistance (par exemple, selon le modèle pressiométrique du Fascicule 62-V $\mathrm{R}_{\text {.an }}$ ), on définit l'erreur de modèle par le rapport $R_{c \text {, mes }} / R_{c \text {, cal }}=\eta$.

La variable aléatoire (c erreur de modèle » est notée $\hat{\eta}$. Ses moments peuvent être estimés par les statistiques classiques sur un jeu de réalisations de $\hat{\eta}$. Ceci ouvre la voie à un calage probabiliste du coefficient de modèle $\gamma_{\text {Rd }}$ par référence à un niveau de fiabilité cible donné. Ce coefficient de modèle devra être utilisé conjointement avec le coefficient partiel ( de valeur ») issu du code ou du règlement de calcul $\gamma$, pour déterminer la valeur de calcul de la résistance.

Ici, l'erreur de modèle $\eta$ est estimée par comparaison entre la résistance calculée qui utilise les pressions limites pressiométriques du sol reconnues spécifiquement au droit de chaque pieu et la résistance mesurée par les essais de pieux: ceci permet, pour l'interprétation de $\eta$, de s'affranchir des incertitudes afférentes à la variabilité spatiale des paramètres d'entrée du modèle, couvertes par ailleurs par les coefficients partiels de valeur comme on vient de le voir.

La loi de $\hat{p}$ est, dans le cadre de cet article, évaluée à partir de la loi de distribution des pressions limites pressiométriques du sol en tenant compte du phénomène de réduction de variance le long du pieu et, de manière plus modérée, en pointe du pieu (effet de moyenne): cela implique que la variabilité de la résistance du pieu soit plus faible que la variabilité des pressions limites pressiométriques elles-mêmes (Bauduin, 2003b).

On doit aussi rajouter la réduction de variance due à la raideur de la structure (transfert des charges d'un pieu ( faible ») vers un pieu ( fort »). Dans la suite de cet article, cet effet n'est pas pris en compte; les coefficients partiels de résistance et de modèle calés avec la fourchette de coefficients de variation infra sont a priori 
valables pour des structures flexibles pour laquelle la ruine d'un pieu ou d'un groupe de pieux très proches signifie la ruine de l'ouvrage.

On suppose que la loi de distribution des pressions limites pressiométriques (paramètre local) est de moyenne $\mu=1,0$ et de coefficient de variation $V=\sigma / \mu$ variant de 0,30 à 0,50 . Avec la réduction de variance supposée d'un facteur $\sqrt{5}$ à $\sqrt{10}$ le long du pieu (de longueur de 10 à 30 m typiquement, avec un sol dont les propriétés seraient moyennement corrélées sur la verticale), sans réduction de variance d'un pieu à l'autre - fondation sur pieux indépendants, nous posons que la loi de distribution de la résistance $\hat{p}$ (paramètre étendu) est de moyenne $\mu_{p}=1,0$ et de coefficient de variation $V_{p}$ variant de 0,10 à 0,20 . Les moments de la loi normale associée $\ln (\hat{\mathrm{p}})$ varient de $\mu_{p}^{\prime}=-0,005$ à $-0,002$ et $\sigma_{p}^{\prime}$ de 0,1 à 0,198.

La résistance à la compression des pieux calculée selon le modèle pressiométrique peut alors être représentée par une variable aléatoire $\mathrm{R}_{\mathrm{c}}$ comme ci-après :

$$
\hat{\mathrm{R}}_{\mathrm{c}}=\hat{\eta} \cdot \hat{\mathrm{p}} \cdot \mathrm{R}_{0}
$$

où :

- $\hat{\eta}$ est la variable aléatoire d'erreur de modèle, supposée lognormale, dont la moyenne et l'écart type doivent être estimés à partir de $\mathrm{N}$ données ;

- $\hat{p}$ est la variable aléatoire représentant l'incertitude sur la résistance du pieu provenant des données pressiométriques, supposée elle aussi lognormale ;

- $R_{0}$ est la valeur moyenne déterministe de $R_{c, \text { cal }}$ destinée à ( centrer » la variable aléatoire $\hat{\mathrm{p}}$.

On suppose aussi pour simplifier que $\mathrm{R}_{0}$ est égal à 1,00.

A noter, au passage, que si l'erreur de modèle était estimée par comparaison avec une résistance calculée avec des pressions limites pressiométriques du sol non reconnues spécifiquement au droit de chaque pieu, alors cette variable aléatoire $\eta^{\prime}$ incluerait aussi les incertitudes afférentes à la variabilité spatiale des paramètres d'entrée du modèle, et la résistance à la compression des pieux devrait être représentée par:

$$
\hat{R}_{c}=\hat{\eta} \cdot R_{0}
$$

C'est la formulation du (42a) qui est mise en œuvre dans la suite de cet article.

Le type de lois supposé a priori pour les deux variables aléatoires $\hat{\eta}$ (erreur de modèle) et $\hat{p}$ (résistance du pieu) est lognormale, non parce qu'un test statistique aurait orienté le choix vers ce type de loi plutôt qu'un autre, mais parce que celui-ci rend les calculs analytiques possibles et plus simples (le produit de deux lois lognormales est une loi lognormale), et qu'il n'est pas réfuté dans le jeu de données qui sert ici de support aux applications numériques. Les variables aléatoires $\ln (\hat{\eta})$ et $\ln (\hat{p})$ suivent alors des lois normales (on parle de loi normale associée) dont les deux premiers moments sont notés ici $\mu^{\prime}$ et $\sigma^{\prime}$. On a les relations suivantes entre les moments d'une loi lognormale $(\mu, \sigma)$ et de la loi normale associée $\left(\mu^{\prime}, \sigma^{\prime}\right)$ :

$$
\begin{aligned}
\mu^{\prime} & =\ln (\mu)-\sigma^{\prime 2} / 2 \\
\sigma^{\prime 2} & =\ln \left(1+\sigma^{2} / \mu^{2}\right)
\end{aligned}
$$

On estime les deux premiers moments de $\hat{\eta}$ par la réalisation des estimateurs classiques de la moyenne et de la variance sur le jeu des données, sans prendre en compte d'incertitude statistique liée à la taille de l'échantillon utilisé.

Les exemples numériques qui suivent sont basés sur 42 essais de pieux (Fig. 1) rassemblés par Renault
(1996) et cités par Frank (1997). On trouve: $m_{n}=1,25$, $s_{\eta}=0,367$ (Fig. 2). La loi lognormale est acceptable avec un niveau de signification supérieur à $90 \%$ selon le test du $\chi^{2}$. Les moments de la loi normale associée $\ln (\hat{\eta})$ sont $\mathrm{m}_{\eta}^{\prime}=0,182, \mathrm{~s}_{\eta}^{\prime}=0,288$.

En éliminant la valeur expérimentale la plus élevée qui se détache si nettement du peloton qu'on la soupçonnerait d'être une valeur aberrante, on trouve: $\mathrm{m}_{\eta}=1,22, \mathrm{~s}_{\eta}=0,266$, les moments de la loi normale associée étant $\mathrm{m}_{\eta}^{\prime}=0,177, \mathrm{~s}_{\eta}^{\prime}=0,215$.

Ces valeurs sont à considérer comme des ordres de grandeur: reposant sur un nombre faible de données quoique statistiquement significatif, elles ne peuvent servir de base à un calage de nature normative ou réglementaire.

Le modèle - ici à partir des essais pressiométriques de sol - semblerait globalement sécuritaire (biais $m_{n}=1,22$ ou $1,25>1$ ). Toutefois, on ne pourrait pas se permettre de corriger le modèle en abattant systématiquement de $25 \%$ la sécurité globale issue du Fascicule $62-\mathrm{V}$, car la dispersion des résultats expérimentaux représentée par l'écart type $s_{\eta^{\prime}}$ fait que le modèle pour-

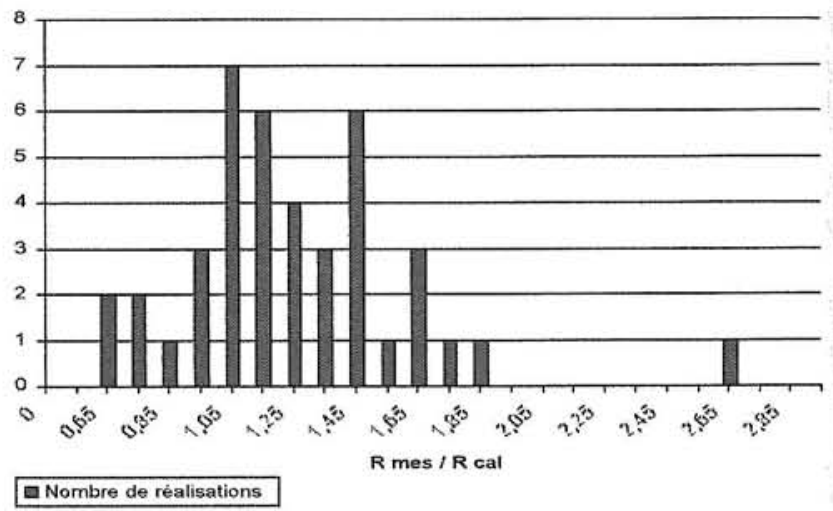

Valeurs de $R_{c, \text { mes }} / R_{c, \text { cal }}=\eta$ pour 42 essais de chargement statique de pieux dans le cas des règles pressiométriques du Fascicule 62-V (Renault, 1996).

Values of $R_{c \text { mes }} / R_{c \text { cal }}=\eta$ for 42 pile static load tests in the case of the pressuremeter rules of Fascicule 62-V (Renault, 1996).

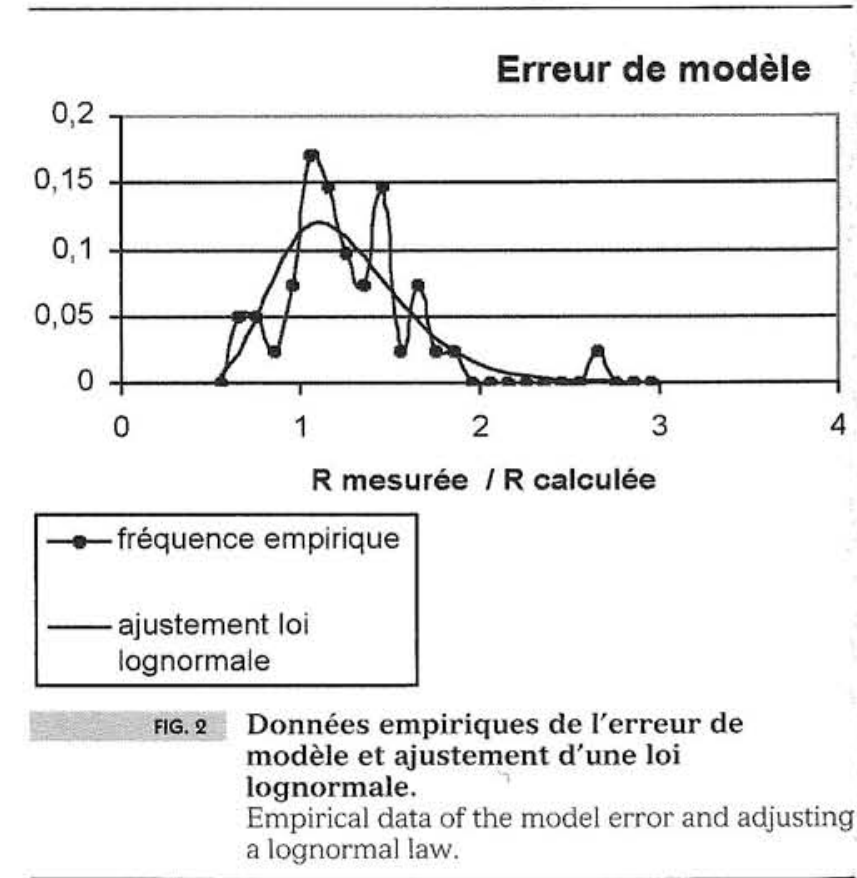


rait aussi manifester une certaine probabilité de se révéler moins sécuritaire que de $25 \%$, voire même de se retrouver optimiste !

De ces deux tendances opposées constatées sur ces 42 pieux (mais qui sont emblématiques de la démarche de calage probabiliste des coefficients partiels), l'une à l'augmentation du niveau de sécurité du modèle pressiométrique (biais > 1), l'autre à la diminution (conséquence de la dispersion s), laquelle va l'emporter? Il revient au calage probabiliste d'apporter une réponse.

\subsection{9.}

\section{Détermination des coefficients partiels de résistance et de modèle}

\subsubsection{Loi de $\hat{\mathrm{R}}_{\mathrm{c}}$}

Dans la méthode fiabiliste de la sécurité, on détermine la valeur de calcul $R_{c, d}$ de telle sorte que:

$$
\operatorname{Prob}\left(\hat{\mathrm{R}}_{\mathrm{c}}^{\mathrm{c}, \mathrm{d}}<\mathrm{R}_{\mathrm{c}, \mathrm{d}}\right)=\mathrm{p}_{\mathrm{c}}
$$

où $\mathrm{p}_{c}$ est la valeur limite de la probabilité admissible de dépassement de la résistance de calcul, qui, selon la théorie de la fiabilité, est reliée à la probabilité admissible de ruine. On peut aussi la représenter par la quantité $u_{d}=\Phi^{-1}\left(p_{c}\right)$ où $\Phi$ est la fonction de répartition de la loi normale « centrée réduite $)$ (c'est-à-dire la loi normale de moyenne nulle et d'écart-type égal à un).

Avec les hypothèses ci-dessus sur $\hat{p}$ et $\hat{\eta}$, la résistance $\mathrm{R}_{\mathrm{c}}$ suit également une loi lognormale dont on peut estimer les deux premiers moments (on note la moyenne $\mu_{\mathrm{Rc}}$ et l'écart type $\sigma_{\mathrm{RC}}$ ). Les moments de la loi normale associée $\ln \left(\hat{R}_{c}\right)$ sont notés $\mu_{R c}^{\prime}$ et $\sigma_{R c}^{\prime}$.

$$
\begin{aligned}
& \mu_{\mathrm{Rc}}^{\prime}=\mu_{\eta}^{\prime}+\mu_{\mathrm{p}}^{\prime} \\
& \sigma_{\mathrm{Rc}}^{\prime 2}=\sigma_{\eta}^{\prime 2}+\sigma_{\mathrm{p}}^{\prime 2}
\end{aligned}
$$

Avec les 42 essais de pieux (non corrigés d'une éventuelle valeur aberrante) on calcule les deux premiers moments de la loi de la résistance (lognormale) produit de la loi des pressions limites pressiométriques et de la loi des erreurs de modèle: $\mu_{\mathrm{Rc}}^{\prime}$ varie de 0,177 à 0,162 et $\sigma_{\mathrm{Rc}}^{\prime}$ varie de 0,304 à 0,349; pour la loi lognormale $\mu_{\mathrm{Rc}}=1,25$ et $\sigma_{\mathrm{Bc}}$ varie de 0,389 à 0,45 . Ces intervalles proviennent de la fourchette de valeurs retenue pour le coefficient de variation des pressions limites pressiométriques $(0,3$ à 0,5$)$ qui se traduisent, avec réduction de variance, par la fourchette $\left(\mathrm{V}_{\mathrm{p}}=0,1\right.$ à 0,2$)$ pour la résistance.

\subsubsection{Sans prise en compte de l'incertitude statistique} sur l'estimation de la loi de l'erreur de modèle

En estimant de façon simplifiée $\mu_{n}^{\prime}$ par $\mathrm{m}_{\eta}^{\prime}$ et $\sigma_{\eta}^{\prime}$ par $\mathrm{s}_{\eta}^{\prime}$ (la formulation rigoureuse pour $\mathrm{R}_{\mathrm{c}, \mathrm{d}}$ est présentée plus loin) on calcule :

$$
R_{c, d}=R_{0} \cdot \exp \left(\mu_{R c}^{\prime}+u_{d} \cdot \sigma_{R c}^{\prime}\right)
$$

où $u_{d}$ prend des valeurs négatives et représente le nombre d'écarts types à retrancher à la moyenne de la loi normale associée pour obtenir la valeur de calcul de la résistance.

De $R_{c, d}$ l'on déduit $\gamma_{\mathrm{R} \text {, calé }}$ (autrement dit le coefficient partiel sur la résistance, à la probabilité cible $\mathrm{p}_{c^{\prime}}$ incluant les incertitudes sur le modèle de résistance et sur la valeur des paramètres d'entrée du modèle) :

avec:

$$
\gamma_{\mathrm{R}, \text { calé }}=\mathrm{R}_{\mathrm{c}, \mathrm{k}} / \mathrm{R}_{\mathrm{c}, \mathrm{d}}
$$

$$
R_{c, k}=R_{0} \cdot \exp \left(\mu_{p}^{\prime}+u_{k} \cdot \sigma_{p}^{\prime}\right)
$$

où $u_{k}$ dépend de la probabilité de dépassement $p_{\text {car }}$ intervenant dans la définition de la valeur caractéristique $\mathrm{p}_{\mathrm{k}}$. Cette quantité prend des valeurs négatives et représente le nombre d'écarts types à retrancher à la moyenne de la loi normale associée pour obtenir la valeur caractéristique de la résistance.

Comme la valeur moyenne de la loi lognormale est différente de la médiane (fractile à $50 \%$ ), nous prendrons $R_{c, k}=R_{0}$ toutes les fois que nous supposerons que la valeur caractéristique est identique à la valeur moyenne. La formule (49) sera en revanche appliquée rigoureusement pour $\mathrm{u}_{\mathrm{k}} \neq 0$.

Remarque: une méthode plus exacte ferait apparaître dans la formule (49) le nombre d'essais de sol sur lesquels les paramètres de la loi de distribution des propriétés de sol auront été estimés.

Puis on déduit le coefficient de modèle de résistance $\gamma_{\text {Rd, calé }}$ par:

$$
\gamma_{\mathrm{Rd} \text {, calé }}=\gamma_{\mathrm{R} \text {, calé }} / \gamma_{\mathrm{t}}
$$

où $\gamma$. est le coefficient partiel de valeur dans la combinaison fondamentale (1,40 dans le cas du Fascicule 62V, et 1,10 dans le cas de l'EN 1997-1, approche 2).

5.3.2.3 Avec prise en compte de l'incertitude statistique sur l'estimation de la loi de l'erreur de modèle

Dans une approche rigoureuse, on doit prendre en compte l'incertitude statistique sur l'évaluation de la loi de l'erreur de modèle à partir d'un nombre limité d'essais (N), la valeur de calcul de la résistance $R_{c, d}$ apparaissant dans l'expression (47) doit être réécrite de manière suivante (annexe D de l'EN 1990 Eurocode: Bases du calcul des structures, CEN 2003) :

$$
R_{c, d}=R_{0} \cdot \exp \left(\mu^{\prime}{ }_{R c}+u_{d} \cdot\left(\sigma^{\prime 2} p / \sigma_{R c}^{\prime}\right)+t_{p_{c}}^{(N-1)} \cdot \sqrt{(1+1 / N)} \cdot\left(s^{\prime 2} / \sigma_{R c}^{\prime}\right)\right)(51)
$$

où $t_{p c}^{(N-1)}$ est le fractile à $p_{c}$ de la loi de Student à N-1

degrés de liberté. Il prend des valeurs négatives et, multiplié par $\sqrt{(1+1 / N)}$, représente le nombre d'écartstypes (en fait, ici la part de l'écart-type total qui a été estimé sur les données) à retrancher à la moyenne de la loi normale associée pour obtenir la valeur de calcul de la résistance.

La relation (49) pour $\mathrm{R}_{\mathrm{c}, \mathrm{k}}$ et le reste s'ensuivent sans changement.

\section{3 .3}

\section{Discussion sur la valeur caractéristique à considérer dans les calages}

Il a été remarqué plus haut que la valeur non pondérée des paramètres de sol était dans la pratique plutôt proche d'une valeur moyenne que l'on aurait un peu rabattue du côté de la sécurité.

Une pratique géotechnique consiste à retrancher à la moyenne un demi à un écart-type (soit $\mathrm{u}_{\mathrm{k}}=-0,5$ à -1 avec une loi normale).

Dans la suite on propose une étude de sensibilité considérant une valeur caractéristique variant en fourchette entre la valeur moyenne: $u_{k}=0$, et le fractile à $5 \%: u_{k}=-1,64$. 


\section{3.}

\section{Détermination de la probabilité cible $p_{c}$ ou du paramètre " $u_{d}$ "}

Le problème est maintenant de déterminer la valeur de la probabilité cible $\mathrm{p}_{\mathrm{c}}$ qui intervient dans la détermination du $\gamma_{\mathrm{R} \text {, calé }}$

Les considérations qui suivent ne s'appliquent qu'à la combinaison type fondamentale, utilisée pour vérifier les états limites relevant de la catégorie des états limites ultimes dans les situations de projet durables et transitoires, pour laquelle les éléments de nature probabiliste ont été développés. S'agissant de la combinaison accidentelle, nous suggérons de s'en tenir aux calages du $\S 5.2$ en raison de la grande variation des niveaux de sécurité pour les combinaisons accidentelles, qui sont commandés essentiellement par le choix de la valeur nominale de l'action accidentelle considérée.

\subsubsection{Méthode des valeurs de calcul}

La méthode des « valeurs de calcul » présentée dans l'annexe C de l'EN 1990 propose:

$$
\begin{gathered}
\mathrm{p}_{\mathrm{c}}=\Phi\left(\mathrm{u}_{\mathrm{d}}\right) \\
\mathrm{u}_{\mathrm{d}}=\alpha_{\mathrm{R}} \cdot \beta
\end{gathered}
$$

où : $\alpha_{\beta}=-0,8$ est la valeur recommandée du coefficient d'influence de la résistance dans la condition d'état limite;

$\beta=3,8$ est l'indice de fiabilité cible recommandé pour la non-occurrence des états limites ultimes en situations de calcul durables ou transitoires.

Il en ressort $u_{d}=-3,04$ ou encore $p_{c}=1,18 \cdot 10^{-3}$

\subsubsection{Méthode inverse}

De nombreux auteurs ont constaté que cette valeur de $u_{d}$ conduisait à des valeurs de coefficients partiels calés élevés comparés à la pratique usuelle. Des travaux de codifications ont été menés en acceptant des valeurs de $\beta$ inférieures: Paikowski (2002) recommande 3,0 pour des fondations sans capacité de redistribution et pour des coefficients de variation de la résistance de 0,3 à 0,4 ; le Code national de la construction au Canada (NBCC) a été calibré pour $\beta=3,5$ (Becker, 1996); les coefficients partiels de la norme hollandaise NEN 6743 ont été établis pour atteindre $\beta=3,4$ et sont valides pour un coefficient de variation de la résistance inférieur à 0,2 (van Tol, 1994).

Une méthode (c inverse » peut alors être tentée pour évaluer la probabilité cible $\mathrm{p}_{c}$ sous-jacente aux valeurs des coefficients partiels existants dans l'EN 1997-1 et dans le Fascicule 62-V (Bauduin, 2003a). Elle consiste à calculer l'indice de fiabilité d'une condition d'état limite de la forme $E<R$, sous des hypothèses concernant le coefficients de variation de l'effet des actions $V_{E}$, le coefficient de variation de la résistance $V_{R}$ et le rapport des moyennes $\mu_{\mathrm{R}} / \mu_{\mathrm{E}}$, celui-ci étant à évaluer pour un ouvrage dimensionné avec les coefficients partiels donnés dans les codes de calcul. On reprend pour cela les valeurs du coefficient global FS' calculé dans la partie 4 (méthode des essais de sol), en se limitant aux situations durables ou transitoires (combinaisons fondamentales). Pour les situations accidentelles, voir la remarque sous 5.3.5.2.

Considérons la relation suivante pour l'indice de fiabilité $\beta$ (une relation un peu différente est proposée dans les Eurocodes qui imposent les valeurs des coeffi- cients $\alpha_{\mathrm{B}}$ et $\alpha_{\mathrm{E}^{\prime}}$, sans incidence notable au regard des buts recherchés) :

$$
\begin{aligned}
& \beta=\left(\operatorname{Ln}\left(\mu_{R} / \mu_{E}\right)+0,5 * \operatorname{Ln}\left(\left(1+V_{E}^{2}\right) /\left(1+V_{R}{ }^{2}\right)\right) /\right. \\
& \left(\sqrt{\operatorname{Ln}}\left(\left(1+V_{E}{ }^{2}\right) /\left(1+V_{R}{ }^{2}\right)\right)\right)
\end{aligned}
$$

où: $V_{E}$ est pris égal à 0,20 pour les combinaisons fondamentales (on choisirait 0,05 pour des actions permanentes et 0,3 à 0,5 pour des actions variables); $\mathrm{V}_{\mathrm{R}}$ est pris égal à $\sigma_{\mathrm{Rc}} / \mu_{\mathrm{Rc}}$ soit 0,311 à 0,36 ;

$\mu_{\mathrm{R}} / \mu_{\mathrm{E}}$ est calculé pour prendre en compte:

- le coefficient partiel sur l'effet des actions (relation $\mathrm{E}_{\mathrm{d}}-$ $\left.E_{k}\right)$ : on choisit 1,425 cohérent avec un cas de charge à $50 \%$ d'actions permanentes et $50 \%$ d'actions variables; - la sécurité liée à la valeur caractéristique des actions (relation $\mathrm{E}_{\mathrm{k}}-\mu_{\mathrm{E}}$ ): on retient ici forfaitairement 1,15; - le coefficient partiel sur la résistance du pieu (relation $R_{d}$ - $\mathrm{R}_{\mathrm{k}}$ ): 1,10 pour l'EN 1997-1 et 1,40 pour le Fascicule 62-V; - la sécurité liée à la valeur caractéristique des résistance (relation $R_{k}-R_{0}$ ): on retient ici forfaitairement 1,05 pour tenir compte de l'abattement effectué souvent dans la pratique par rapport à la valeur moyenne; - le biais du modèle de résistance (relation $R_{0}-\mu_{E}$ ): $\mu_{\mathrm{Rc}}=1,25$.

Le rapport $\mu_{\mathrm{R}} / \mu_{\mathrm{E}}$ est ainsi calculé à 2,37 pour l'EN 1997-1 et 3,01 pour le Fascicule 62-V.

Le calcul donne un indice de fiabilité sous-jacent relativement faible: $\beta$ varie entre 2,04 et 2,3 pour l'EN 1997-1, entre 2,64 et 2,97 pour le Fascicule 62-V.

A titre d'étude de sensibilité, le même type de calcul mené avec les données d'erreur de modèle excluant la valeur extrême soupçonnée être aberrante et avec un coefficient de variation intermédiaire pour la résistance $\mathrm{V}_{\mathrm{p}}=0,125$, donne un coefficient de variation réduit $\mathrm{V}_{\mathrm{B}}$ de 0,251 et, en contrepartie, un biais réduit de 1,22 . Il est intéressant de noter que cela fait augmenter $\beta$ significativement, qui vaut alors 2,61 pour l'EN 1997-1, et 3,37 pour le Fascicule 62-V. Ceci montre l'importance de la qualité de la base de données utilisée pour les calages, ainsi que du jeu d'hypothèses sous-jacentes.

On propose à ce stade d'effectuer une étude paramétrique pour des valeurs de $\beta$ en fourchette entre 2,5 et 3,8 soit $u_{d}$ variant entre $-2,0$ et $-3,04$.

Pour les calages, on proposera de considérer la valeur cible de $\beta=3,0$, soit $u_{d}=2,4$.

Enfin, on vérifie que $\sigma_{\mathrm{E}} / \sigma_{\mathrm{R}}=\mathrm{V}_{\mathrm{E}} \cdot \mu_{\mathrm{E}} / \mathrm{V}_{\mathrm{B}} \cdot \mu_{\mathrm{R}}=0,18>$ 0,16 , qui est la condition de validité du coefficient d'influence $\alpha_{\mathrm{R}}$ de 0,8 ci dessus (condition (C7) de l'EN 1990 - annexe C).

5.3.4.3 Apport de la prise en compte de l'incertitude statistique sur l'estimation de la loi de l'erreur de modèle

Dans l'approche rigoureuse prenant en compte l'incertitude statistique sur l'évaluation de l'erreur de modèle, la valeur de calcul de la résistance est déterminée non seule-

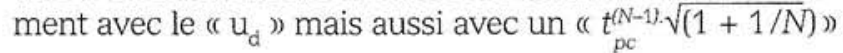

C'est quand ces deux paramètres sont numériquement égaux (ce qui se produit conformément à la théorie pour un nombre infini d'essais, $\mathrm{N}=\infty$ ) que les deux formulations (47) et (51) donnent des résultats identiques pour la valeur de calcul de la résistance.

Cette approche donne des valeurs de ( $\left(u_{d}\right)$ plus sécuritaires que celles issues de la formulation simple ne prenant pas en compte l'incertitude statistique sur 
l'estimation de l'erreur de modèle, et ce d'autant plus que $\left|u_{d}\right|$ augmente.

Dans l'application numérique de cet article (voir plus bas) on met en œuvre tant la formulation simplifiée (47) que la formulation rigoureuse (51). Une rapide étude de sensibilité générale a été effectuée; elle a montré que la formule rigoureuse conduit à des valeurs plus élevées du coefficient $\gamma_{\text {Rd, calé }}$ de $7 \%$ à $12 \%$ par rapport à la formulation simplifiée.

\subsection{5}

\section{Application numérique}

\subsubsection{Coefficient de modèle de résistance -} combinaison fondamentale

L'équation (48) permet de calculer le coefficient de résistance $\gamma_{R \text {, calét }}$

Compte tenu de la sensibilité que manifeste le coefficient $\gamma_{\mathrm{R} \text {, calé }}$ au niveau de sécurité cible de la valeur de calcul, que représente le paramètre $u_{d}$, et selon le choix initial de la valeur caractéristique que représente le paramètre $u_{k}$ on fait figurer la relation entre ces deux grandeurs selon plusieurs hypothèses sur le coefficient de variation de la résistance du pieu $\left(V_{p}\right)$ et selon la valeur caractéristique retenue (Fig. 3).

Le tableau VII présente le $\gamma_{\mathrm{R} \text {, calé }}$ avec un indice de fiabilité cible de 3,0 $\left(u_{d}=-2,4\right)$.

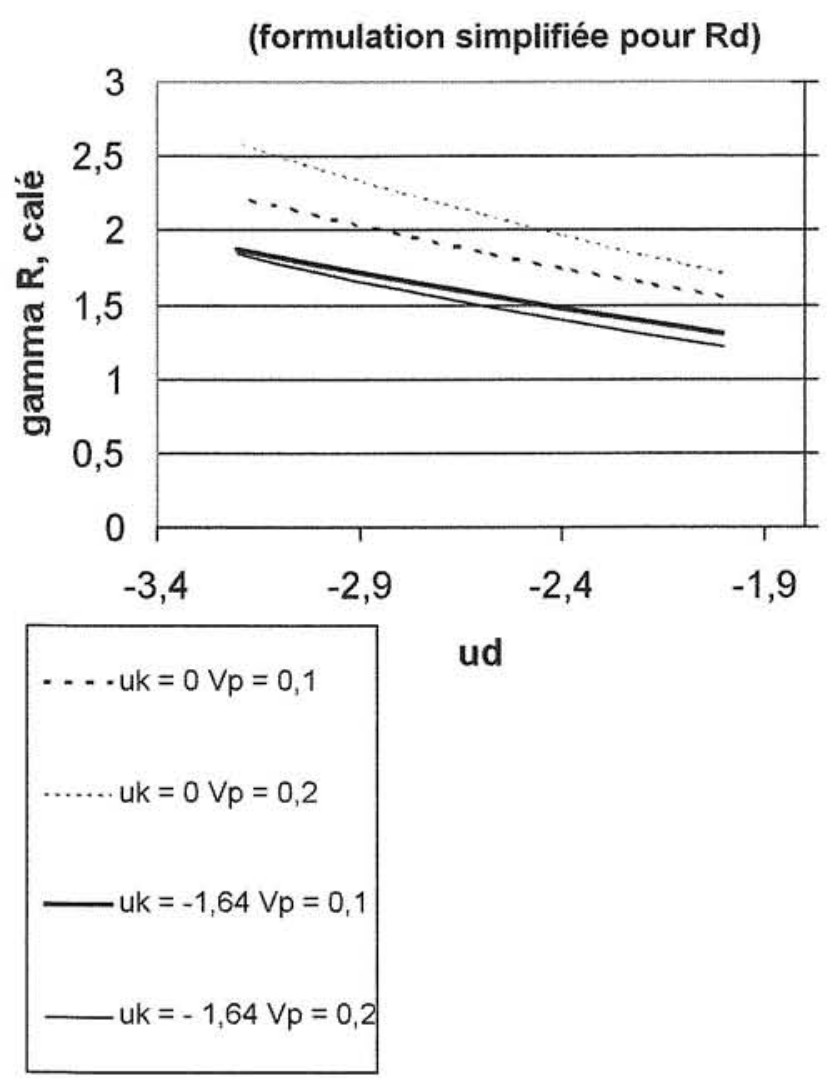

FIG. 3 Sensibilité de $\gamma_{\mathrm{R} \text { calć }}$ au niveau de sécurité cible de la valeur de calcul $u_{d^{\prime}}$ selon plusieurs hypothèses sur le coefficient de variation de la résistance du pieu et la définition de la valeur caractéristique. Sensitivity of $\gamma_{\text {a }}$ with the safety target of the design value $u_{d}$ according to various assumptions on the coefficient of variation of the pile resistance and the definition of the characteristic value.
L'équation (50) permet de calculer le coefficient de modèle de résistance qui est présenté sous les mêmes hypothèses dans le tableau VIII.

Avec la formulation simplifiée qui a servi de base à l'application numérique et un niveau de fiabilité cible $\beta$ de 3,0 , on obtient les valeurs arrondies suivantes: - en supposant une valeur caractéristique proche de la valeur moyenne des propriétés des sols: 1,40 ,

* avec le $\gamma_{\mathrm{t}}$ du Fascicule 62-V: $\gamma_{\text {Rd, calé }}$ entre 1,24 et

* avec le $\gamma_{\mathrm{t}}$ de l'EN 1997-1, approche 2: $\gamma_{\text {Rd calé }}$ entre 1,58 et 1,78 ;

- en supposant une valeur caractéristique proche du fractile à $5 \%$ :

* avec le $\gamma_{t}$ du Fascicule 62-V: $\gamma_{\text {Rd calé }}$ entre 1,00 et 1,05,

* avec le $\gamma_{t}$ de l'EN 1997-1, approche 2: $\gamma_{\text {Rd calé }}$ entre 1,27 et 1,34 .

Le calcul, mené avec la formulation (crigoureuse ») de l'équation (51), et avec:

- les données d'erreur de modèle excluant la valeur extrême soupçonnée d'être aberrante ;

- un coefficient de variation intermédiaire de la résistance $\mathrm{V}_{\mathrm{p}}=0,125$;

- une valeur caractéristique obtenue en retranchant un demi écart type $\left(u_{k}=-0,5\right)$, et

- l'indice de fiabilité cible $\beta=3,0\left(u_{d}=-2,4\right)$, fournit:

$$
\begin{aligned}
& \gamma_{\mathrm{R}, \text { calé }}=1,548 \\
& \gamma_{\mathrm{Rd}, \text { calé }}=1,407 \text { pour l'EN 1997-1 } \\
& \gamma_{\mathrm{Rd}, \text { calé }}=1,106 \text { pour le Fascicule } 62-\mathrm{V} .
\end{aligned}
$$

\subsubsection{Combinaison accidentelle}

Pour la combinaison accidentelle (hors du champ du calage ci-dessus), il serait nécessaire d'adapter la méthode proposée en introduisant notamment le niveau de sécurité propre à la valeur nominale de l'action accidentelle considérée dans le cas de charge, ou la probabilité d'occurrence de la configuration accidentelle, avec le niveau de sécurité cible propre à la vérification $(\beta)$.

\subsubsection{Coefficient réuni de modèle}

On peut alors calculer le coefficient réuni de modèle $\gamma_{\mathrm{d}, \text { calé }}$ (équation 55) présenté dans le tableau IX:

$$
\gamma_{\mathrm{d}, \text { calé }}=\gamma_{\mathrm{Rd}, \text { calé }}{ }^{*} \gamma_{\mathrm{Sd}}
$$

\section{6}

\section{Conclusion}

Les changements intervenus depuis l'époque de la pré-norme, et la nécessité actuelle de calibrer les raccordements nationaux à l'Eurocode 7, justifient la mise à jour de la comparaison effectuée par Frank (1997).

Pour le dimensionnement des pieux sous charges axiales de compression vis-à-vis de l'état limite de défaut de capacité portante, bien que les approches puissent paraître différentes et qu'il y ait des différences dans les valeurs des coefficients partiels, on confirme que les niveaux globaux de sécurité recommandés par la norme européenne EN 1997-1 et par le règlement officiel français "Fascicule $n^{\circ} 62$ - Titre V ) présentent des ordres de grandeur comparables, le Fascicule $62-\mathrm{V}$ étant cependant dans l'ensemble plus 
TABLEAU VII Coefficient partiel $\gamma_{\mathrm{R} \text {, calé }}$ (modèle des essais de sol) Exemple numérique basé sur 42 essais. Partial factor $\gamma_{R^{\prime}}$ calib (ground tests model). Numerical example based on 42 tests.

\begin{tabular}{|c|c|c|c|c|}
\hline \multirow{2}{*}{$\begin{array}{l}\text { Définition de la valeur caractéristique } \mathrm{p}_{\mathrm{k}} \\
\text { Choix du coefficient de variation de } \hat{\mathrm{p}}\end{array}$} & \multicolumn{2}{|c|}{ Valeur moyenne } & \multicolumn{2}{|c|}{ Fractile à $5 \%\left(u_{k}=-1,64\right)$} \\
\hline & 0,10 & 0,20 & 0,10 & 0,20 \\
\hline $\mathrm{u}_{\mathrm{d}}$ & \multicolumn{4}{|c|}{$-2,4$} \\
\hline$\frac{a}{R_{c, k} / R_{0}}$ & \multicolumn{2}{|c|}{1,00} & 0,845 & 0,709 \\
\hline$\frac{R_{c, d} / R_{0}}{2}$ & 0,575 & 0,509 & 0,575 & 0,509 \\
\hline$\gamma_{\mathrm{R}, \text { calé }}$ & 1,739 & 1,965 & 1,470 & 1,393 \\
\hline
\end{tabular}

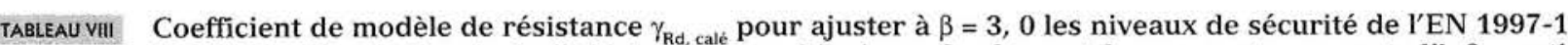
(approche 2) et du Fascicule 62-V dans les combinaisons fondamentales prenant en compte l'information supplémentaire sur l'erreur de modèle (modèle des essais de sol).

Resistance model factor $\gamma_{\text {Rd, caili }}$ to adjust to $\beta=3.0$ the safety levels of EN 1997-1 (approach 2) and Fascicule 62-V for the fundamental combinations taking account of supplementary information on model error (ground tests model).

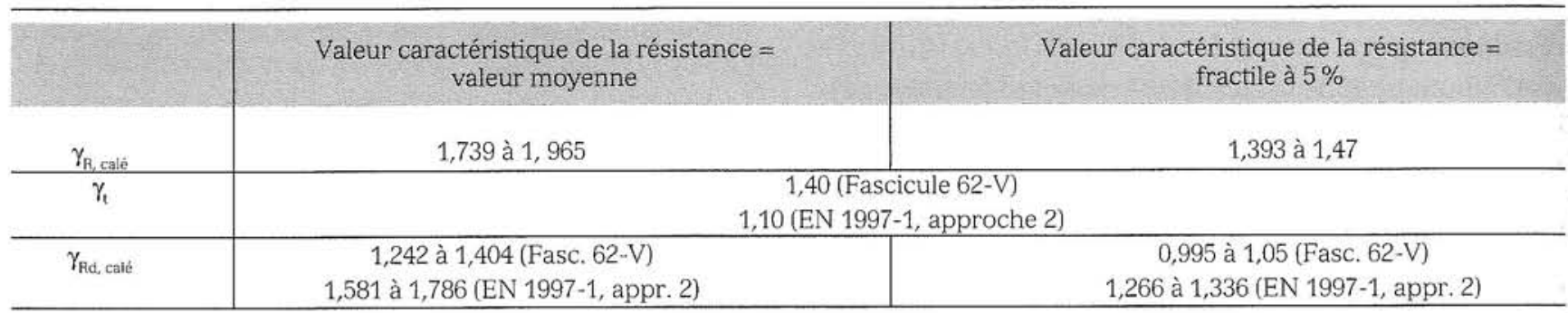

TABLEAU IX Coefficient réuni de modèle $\gamma_{\text {d calé }}$ pour ajuster à $\beta=3,0$ les niveaux de sécurité de l'EN 1997-1 (approche 2) et du Fascicule 62-V dans les combinaisons fondamentales prenant en compte l'information supplémentaire sur l'erreur de modèle (modèle des essais de sol).

Unified model factor $\gamma_{\text {d. calib }}$ to adjust to $\beta=3.0$ the safety levels of EN 1997-1 (approach 2) and Fascicule 62-V for the fundamental combinations taking account of supplementary information on model error (ground tests model).

\begin{tabular}{c|c|c}
\hline & $\begin{array}{c}\text { Valeur caractéristique de la résistance } \\
\text { valeur moyenne }\end{array}$ & $\begin{array}{c}\text { Valeur caractéristique de la résistance }= \\
\text { fractile à 5\% }\end{array}$ \\
$\gamma_{\text {Rd, calé }}$ & \begin{tabular}{c}
1,242 à 1,404 (Fasc. 62-V) \\
0,995 à 1, 05 (Fasc. 62-V) \\
\hline$\gamma_{\text {Sd }}$
\end{tabular}$\quad$\begin{tabular}{c|c}
1,581 à 1, 786 (EN 1997-1, appr. 2) \\
1,266 à 1,336 (EN 1997-1, appr. 2)
\end{tabular} \\
\hline$\gamma_{\text {d, calé }}$ & 1,397 à 1, 579 (Fasc. 62-V) & 1,125 \\
\hline
\end{tabular}

sécuritaire. Dans le cas des essais de pieux, les différences sont plus notables, pour les exemples traités ici, lorsque l'on dispose de plusieurs essais de chargement statique.

La méthode de calage probabiliste, qui a été bâtie ici pour l'approche 2 de l'EN 1997-1, permet de déboucher sur un ordre de grandeur du coefficient de modèle de résistance $\gamma_{\mathrm{Rd}}$ qui, utilisé conjointement avec les coefficients partiels de l'EN 1997-1 et pour peu que l'on reste bien dans les ordres de grandeur de variabilité des principaux paramètres explicités plus haut, permet de retrouver les niveaux de sécurité du Fascicule 62-V. Dans la méthode, ces niveaux de sécurité peuvent être corrigés facilement pour tenir compte de toute comparaison entre un modèle basé sur des essais de sol et une banque de donnée d'essais de chargement statique de pieux. Pour le modèle à partir d'essais pressiométriques du Fascicule 62-V, le calage probabiliste des coefficients partiels de résistance a été mis en œuvre à l'aide d'une série d'essais de pieux en nombre limité pour l'instant $(\mathrm{N}=42)$. La qualité et la représentativité de la base de données sont bien évidemment de première importance. Les questions qui se sont posées relèvent principalement de trois ordres:
- quel est le niveau de sécurité cible de la valeur caractéristique des propriétés des sols? Les deux cas extrêmes ont été traités selon que la valeur caractéristique est réputée être la valeur moyenne ou le fractile à $5 \%$ de la loi de distribution supposée de la propriété géotechnique, mais normalement on devrait tenir compte aussi du nombre d'essais effectués;

- quel est le niveau de sécurité cible de la valeur de calcul de la résistance géotechnique (combinaison fondamentale)? Il apparaît que la méthode des (c valeurs de calcul ») de l'annexe C de l'EN 1990 (CEN, 2003) donne des résultats très sécuritaires au regard de la pratique. On a mis en œuvre une « méthode inverse ») qui calcule une probabilité cible de ruine sous-jacente, en référence aux coefficients partiels recommandés par l'Eurocode 7 et par le Fascicule 62-V;

- quelle est l'origine des variations constatées de la comparaison entre résistances calculées et mesurées? La méthode de calage suppose en effet que ces variations représentent seulement l'incertitude de modèle et non les incertitudes sur les paramètres des sols entrés dans le modèle de calcul. On a admis ici que les calculs d'incertitude de modèle reposaient sur des données d'essais de pieux avec des valeurs correctes des para- 
mètres d'entrée des sols, et en supposant que la dispersion due à l'exécution des pieux était comprise dans le coefficient ( de valeur »).

En résumé, dans le cas où des essais de pieux sont exécutés, en se basant sur l'ébauche d'étude de sensibilité de cet article (calage par ajustement), on pourrait retenir typiquement des valeurs de $\gamma_{\text {Rd }}$ entre 1,10 et 1,30 (combinaison fondamentale), entre 1,00 et 1,25 (combinaison accidentelle).

Pour le cas où l'on utilise les résultats d'essais de sol, dans la combinaison fondamentale, avec les données limitées qui ont été traitées ici, pour un niveau de fiabilité cible $\beta$ de 3,0 et en considérant que la valeur caractéristique des propriétés des sols s'écarte légèrement de la valeur moyenne (à laquelle on retrancherait un demi écart type, valeur à moduler selon le nombre d'essais préalables effectués), et en prenant le coefficient de variation de la résistance $V_{p}=0,125$, on pourrait retenir des valeurs arrondies suivantes de $\gamma_{\mathrm{Pd} \text {, calé }}$ : $\gamma_{\text {Rd. calé }}=1,10$ quand il est utilisé avec $\gamma_{\mathrm{t}}=1,40 \mathrm{du}$ Fascicule $62-\mathrm{V}$;

$\gamma_{\text {Rd, calé }}=1,40$ quand il est utilisé avec $\gamma_{t}=1,10$ de $l^{\prime} E N$ 1997-1, approche 2.

Ces valeurs sont à considérer comme des ordres de grandeur; reposant sur un nombre faible de données quoique statistiquement significatif, elles ne peuvent servir telles quelles de base à un calage de nature normative ou réglementaire.
Enfin le coefficient réuni de modèle $\gamma_{d}$ donné par état limite est un outil d'usage pratique qui permet d'ajuster au niveau national les niveaux de sécurité par l'intermédiaire d'une seule quantité, sans qu'il soit besoin de ( retoucher ) les coefficients partiels de valeur présents dans le corps du texte des Eurocodes.

Bien évidemment cette méthode reste valable pour un jeu de données différent ou plus complet relatif à l'erreur de modèle. A cette fin on donne ci-après le formulaire complet permettant d'exécuter de nouveaux calculs avec un autre jeu de données. Cette méthode pourrait aussi être adaptée à l'approche des « pieux modèle » préconisée par l’EN 1997-1 (CEN, 2004) et les règles de calcul à partir des résultats d'essais in situ (pressiométriques et pénétrométriques) du Fascicule $62-V$, qui permettent de tenir compte du nombre de profils d'essais de sol disponibles, et aux fondations sollicitant plusieurs pieux avec possibilité de redistribution des efforts des pieux « faibles » aux pieux ( forts ).

\footnotetext{
REMERCIEMENTS

Les auteurs remercient vivement les Professeurs Jean-Louis Favre, Christophe Bauduin et $M$. François Baguelin pour leur aide apportée à la mise au point du présent article.
}

\section{Bibliographie}

AFNOR - Eurocode 7 Calcul géotechnique Partie 1: Règles générale. XP ENV 19971 (P 94-250-1), décembre 1996, $112 \mathrm{p}$. Association française de normalisation (AFNOR), Paris.

Bauduin C. - Design procedure according to Eurocode 7 and analysis of the test results. Proc, of the symp. on screw piles: Installation and design in stiff clay. Balkema, Rotterdam, 2001, p. 275-303.

Bauduin C. - Assessment of model factors and reliability index for ULS design of pile foundations. IVth Conf. on bored and auger piles, Gand, 2003a.

Bauduin C. - Communication privée, 2003b.

Becker D. - Eighteenth Canadian Geotechnical Colloquium. Limit state design for foundations, Part II : Development for the National Builing Code of Canada. Can. geotech. J. 33(6), 1996, p 984-1007.

Bustamante M., Gianeselli L. - Prévision de la capacité portante des pieux isolés sous charge verticale. Règles pressiométriques et pénétrométriques. Bull. Liaison Labo. P. et Ch., n¹13, 1981, p. 83-108.

Bustamante M., Gianeselli L. - Communication privée, 2003.

CEN - EN 1990: Eurocode: Bases du calcul des structures, Comité européen de normalisation (CEN), Bruxelles, Version française: NF EN 1990: 2002, AFNOR, 20 mars 2003, 90 p.
CEN - EN 1997-1: Eurocode 7 Calcul géotechnique. Partie 1. Règles générales (étape 49) Version française (F), Comité européen de normalisation (CEN) Bruxelles, nov. 2004, 174 p.

CETMEF - Rosa 2000, Recommandations pour le calcul aux états limites des ouvrages en site aquatique, Centre d'études techniques maritimes et fluviales (CETMEF), Compiègne, 2001.

Frank R. - The new Eurocode and the new French code for the design of deep foundations. Keynote lecture. Proc. FHWA Int. Conf. Design and Construction of Deep Foundations, Oriando, Florida, 68 December 1994, vol. 1, p. 279-304.

Frank R. - Some comparisons of safety for axially loaded piles. Proc. Int. Seminar Design of Axially loaded piles, European Practice, ERTC3, Brussels, 17-18 April 1997, F. De Cock \& C. Legrand, Eds, p. 39-46, Balkema, Rotterdam. Version française: Comparaison de coefficients de sécurité pour les pieux sous charges axiales. Revue française de géotechnique $n^{\circ} 81,4^{\text {e }}$ trimestre 1997 , p. 27-32.

Kovarik J.-B. - Qu'est-ce qu'un coefficient de sécurité en génie civil? Revue française de génie civil, vol. $4, n^{\circ} 6$, Hermès, Paris, déc. 2000.

Kovarik J.-B. - Méthodes de calage des coefficients de modèle pour les ouvrages maritimes et fluviaux. $2^{e}$ conférence JN-FIAB'98, Hermès, Paris, 1998, p. 29-38.
ISSMFE - Subcommittee on Field and Laboratory Testing. Axial pile loading test. Part 1: Static loading. Geotechnical Testing J. 8(2), 1985, p. 79-90.

Mathieu H, et al., - Manuel de sécurité des structures. Comité européen du béton, bulletins $n^{\circ} 106$ et 107, 1975.

MELT - Règles techniques de conception et de calcul des fondations des ouvrages de génie civil, CCTG, Fascicule $n^{\circ} 62$ - Titre V. Ministère de l'Équipement du Logement et des Transports, Paris, 1993.

Paikowski G.S. - Load and resistance factor design (LRFD) for deep foundations. Foundation design codes in view of international harmonization and perfor mance. IWS Kamakura, Japon, 2002, p. 59-94.

Renault J. - Communication privée (document interne au Groupe d'étude de l'Eurocode 7 GEEC7 de l'AFNOR), 1996.

Tol (van) A.F. - Hoe betrouwbaar is de paalfundering? Intrereede. TU Delft, Faculté de génie civil (cité par Bauduin, 2003b), 1994.

Wastiaux M., Ducroq J., Baguelin F. - Les fondations maritimes. Revue Travaux consacrée au pont Vasco de Gama, $n^{\circ} 743$, juin 1998, p. 33-41.

Wastiaux M., Ducroq J., Corbetta F. - Les pieux maritimes du pont Vasco de Gama. Revue française de géotechnique, $\mathrm{n}^{\circ} 87,2^{\mathrm{e}}$ trimestre 1999, p. 27-33. 


\section{NOTATIONS}

\begin{tabular}{|c|c|c|}
\hline $\mathrm{R}_{\mathrm{c}}$ & : & résistance en compression \\
\hline $\mathrm{R}_{\mathrm{c}, \mathrm{k}}$ & : & valeur caractéristique de la résistance en compression \\
\hline$R_{c, d}$ & : & valeur de calcul de la résistance en compression \\
\hline $\mathrm{R}_{\mathrm{c}, \mathrm{m}}$ & : & valeur moyenne des résistances mesurées \\
\hline $\mathrm{R}_{\mathrm{c}, \max }$ & : & la plus grande des résistances mesurées \\
\hline $\mathrm{R}_{c, \text { mes }}$ & : & valeur mesurée de la résistance prise en compte \\
\hline $\mathrm{R}_{c, \min }$ & $:$ & la plus petite des résistances mesurées \\
\hline $\mathrm{R}_{\mathrm{c}, \mathrm{cal} \mathrm{m}}$ & : & moyenne des valeurs de la résistance calculées \\
\hline $\mathrm{R}_{\mathrm{c}, \mathrm{cal} \text { min }}$ & : & la plus petite des valeurs de la résistance calculées \\
\hline$\xi_{1}, \xi_{2}, \xi^{\prime}, \xi_{3}, \xi_{4}$ & : & coefficients de réduction statistique \\
\hline$R_{b, k}$ & : & valeur caractéristique de la résistance de la pointe \\
\hline$R_{s, k}$ & : & valeur caractéristique de la résistance du fût \\
\hline$q_{b, k}$ & : & résistance de pointe \\
\hline $\mathrm{q}_{\mathrm{s}, \mathrm{k}}$ & : & frottement latéral \\
\hline E & : & effet des actions \\
\hline $\mathrm{F}_{\mathrm{c}}$ & : & actions de compression appliquées en tête du pieu \\
\hline $\mathrm{F}_{c, k}$ & : & valeur caractéristique des actions \\
\hline $\mathrm{F}_{\mathrm{c}, \mathrm{d}}$ & : & valeur de calcul des actions \\
\hline FS & $=$ & 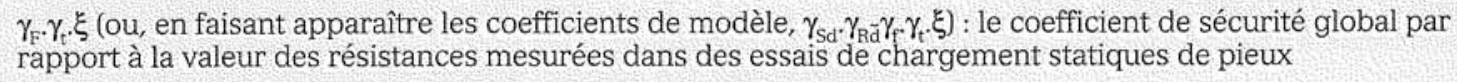 \\
\hline FS' & $=$ & $\begin{array}{l}\gamma_{\mathrm{F}} \cdot \gamma_{t}\left(\mathrm{ou}, \text { en faisant apparaître les coefficients de modèle, } \gamma_{\mathrm{Sd}} \cdot \gamma_{\mathrm{Rad}} \gamma_{\mathrm{t}} \gamma_{\mathrm{t}}\right): \text { le coefficient de sécurité global par } \\
\text { rapport à la valeur caractéristique de la résistance axiale du pieu telle qu'elle est évaluée par la méthode de } \\
\text { prévision }\end{array}$ \\
\hline$\gamma_{\mathrm{F}}$ & : & 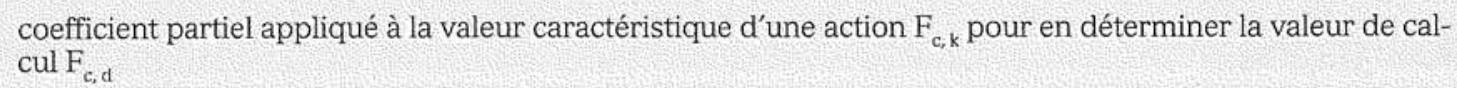 \\
\hline$\gamma_{\mathrm{f}}$ & : & $\begin{array}{l}\text { coefficient partiel appliqué à la valeur caractéristique d'une action pour en déterminer la valeur de calcul, } \\
\text { sans prise en compte des incertitudes de modèle }\end{array}$ \\
\hline$\gamma_{\mathrm{R}}$ & : & $\begin{array}{l}\text { coefficient partiel appliqué à la valeur caractéristique d'une résistance } R_{c, k} \text { pour en déterminer la valeur de } \\
\text { calcul } R_{c, d}\end{array}$ \\
\hline$\gamma_{\mathrm{R}, \text { calé }}$ & : & coefficient partiel de résistance qui résulte d'un processus de calage ou de calibration \\
\hline$\gamma_{\mathrm{r}}$ & : & $\begin{array}{l}\text { coefficient partiel appliqué à la valeur caractéristique d'une résistance pour en déterminer la valeur de cal- } \\
\text { cul, sans prise en compte des incertitudes de modèle }\end{array}$ \\
\hline$\gamma_{\mathrm{t}}$ & : & $\begin{array}{l}\text { coefficient partiel sur la résistance totale, ayant la même signification qu'un } \gamma_{\mathrm{R}^{\prime}} \text { et aussi qu'un } \gamma_{\mathrm{r}} \text { quand on } \\
\text { suppose que } \gamma_{\mathrm{R}}=1,00\end{array}$ \\
\hline$\gamma_{\mathrm{b}}$ & : & coefficient partiel sur la résistance de pointe \\
\hline$\gamma_{\mathrm{s}}$ & : & coefficient partiel sur la résistance de fût \\
\hline$\gamma_{\text {Sd }}$ & : & coefficient de modèle d'actions (noté $\gamma_{\mathrm{F} 3}$ dans les règlements français) \\
\hline$\gamma_{S d, \text { acc }}$ & : & coefficient de modèle d'action, calé pour être utilisé dans la combinaison type accidentelle \\
\hline$\gamma_{\text {Sd, fond }}$ & : & coefficient de modèle d'action, calé pour être utilisé dans la combinaison type fondamentale \\
\hline$\gamma_{\mathrm{Rd}}$ & : & coefficient de modèle de résistance \\
\hline$\gamma_{\mathrm{Rd}, \text { calé }}$ & : & coefficient de modèle de résistance qui résulte d'un processus de calage ou de calibration \\
\hline$\gamma_{\mathrm{Rd}, \text { cale, fond }}$ & : & coefficient de modèle de résistance, calé pour être utilisé dans la combinaison type fondamentale \\
\hline$\gamma_{\mathrm{Rd}, \text { calé, acc: }}$ & : & coefficient de modèle de résistance, calé pour être utilisé dans la combinaison type accidentelle \\
\hline$\gamma_{\mathrm{d}}$ & : & coefficient réuni de modèle défini par le produit $\gamma_{\mathrm{Sd}} \cdot \gamma_{\mathrm{Rd}}$ \\
\hline$\gamma_{\mathrm{d}, \text { cale }}$ & : & coefficient réuni de modèle qui résulte d'un processus de calage ou de calibration \\
\hline$\gamma_{d, \text { calé, fond }}$ & : & coefficient réuni de modèle, calé pour être utilisé dans la combinaison type fondamentale \\
\hline$\gamma_{\mathrm{d}, \text { calé, acc }}$ & : & coefficient réuni de modèle, calé pour être utilisé dans la combinaison type accidentelle \\
\hline
\end{tabular}




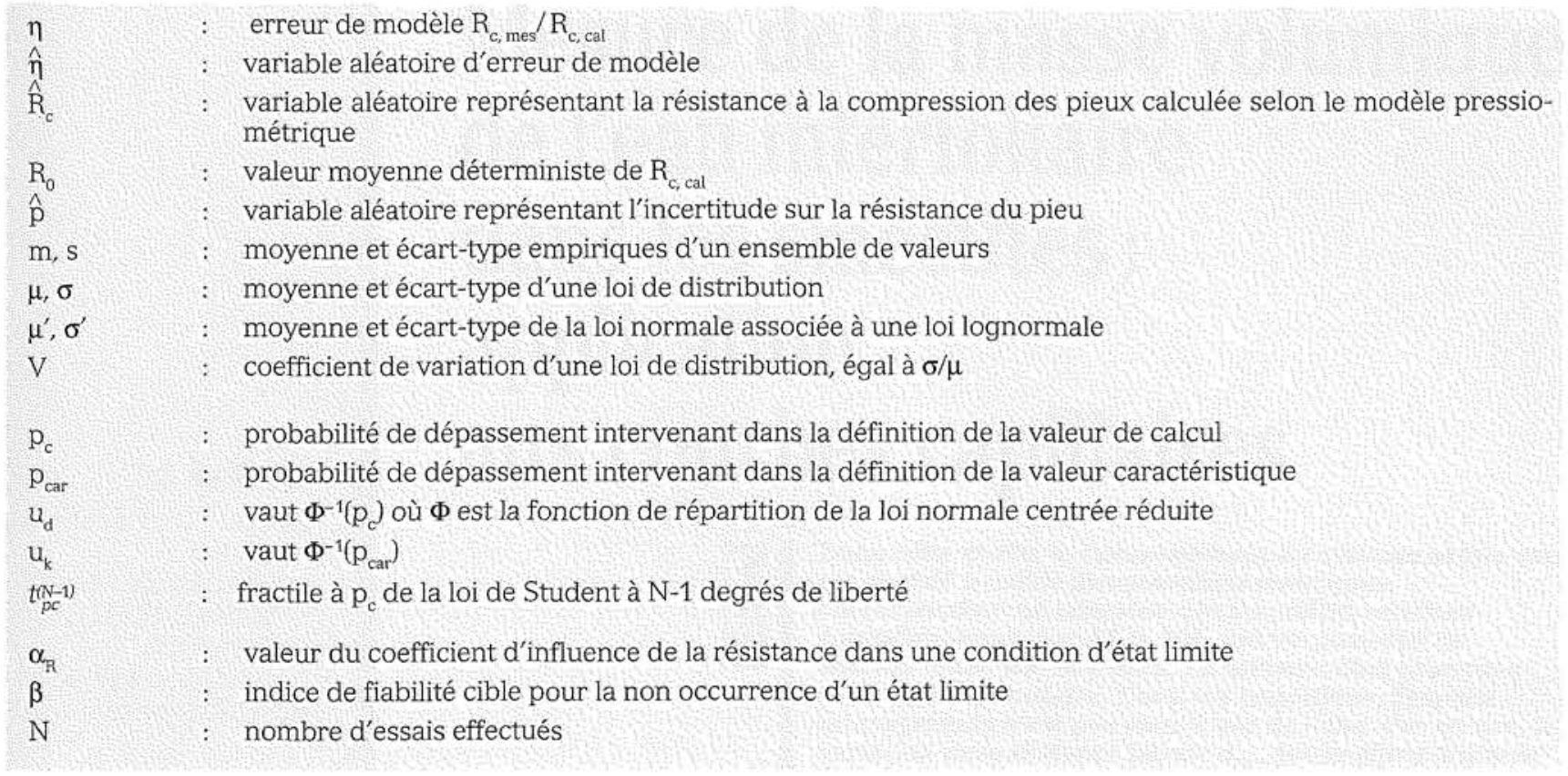

\section{ANNEXE : FORMULAIRE}

$\gamma_{d, \text { cale }}=\gamma_{\text {Rd, cale }}{ }^{*} \gamma_{\text {Sd }}$

$\gamma_{\text {Rd, calé }}=\gamma_{\mathrm{R}, \text { cale }} \gamma_{\mathrm{t}}$

$\gamma_{\mathrm{Sd}}$ est le coefficient de modèle pour les actions (valeur usuelle: 1, 125 dans la combinaison fondamentale, 1, 00 dans la combinaison accidentelle)

$\gamma_{R, \text { calé }}=R_{c, K} / R_{c, d}$

$\gamma_{t}$ est le coefficient partiel de valeur pour la résistance utilisé dans la combinaison fondamentale (Fascicule 62-V, EN 1997-1)

$R_{c, k}=R_{0} \cdot \exp \left(\mu_{p}^{\prime}+u_{k} \cdot \sigma_{p}^{\prime}\right)$

(formulation rigoureuse) :

$R_{C, d}=R_{0} \cdot \exp \left(\mu_{R c}^{\prime}+u_{d} \cdot\left(\sigma^{\prime 2} p / \sigma_{R c}{ }_{R c}\right)+t_{p_{C}}^{(N-1)} \sqrt{(1+1 / N)} \cdot\left(S^{2} \eta / \sigma_{R c}^{\prime}\right)\right)$

(formulation simplifiée):

$R_{c, d}=R_{g} \exp \left(\mu_{R c}^{\prime}+u_{d d^{\prime}} \sigma_{R c}\right)$

$u_{\mathrm{f}}$ est liée à la probabilité de dépassement définissant la valeur de calcul; u. est liée à la probabilité de dépassement définissant la valeur caractéristique (de - 1, 64 pour un fractile à $5 \%$ à 0,00 pour une valeur moyenne).

$$
\begin{aligned}
& \mu_{\mathrm{Rc}}^{\prime}=\mathrm{m}_{\eta \eta}^{\prime}+\mu_{\mathrm{p}}^{\prime} \\
& \sigma_{\mathrm{Rc}}^{\prime 2}=\mathrm{s}^{\prime 2}{ }_{\eta}+\sigma^{\prime 2}{ }_{\mathrm{p}}
\end{aligned}
$$

$\mathrm{m}^{\prime}$ et $\mathrm{s}^{\prime 2}$ sont la moyenne et la variance empiriques de la loi normale $\ln (\hat{\eta})$ (erreur de modèle);

$\mu_{p}^{\prime}$ et $\sigma_{p}^{\prime 2}$ sont la moyenne et la variance de la loi normale $\ln (\hat{p})$ (résistance du pieu)

$\mathrm{N}$ est le nombre d'essais de comparaison pris en compte dans l'estimation de la loi de l'erreur de modèle;

$\mathrm{R}_{0}$ est la valeur moyenne de la loi de probabilité de la résistance sans tenir compte de l'erreur de modèle. $t^{(N-1)}$ est le fractile à $\mathrm{p}_{\mathrm{c}}=\Phi\left(\mathrm{u}_{\mathrm{d}}\right)$ de la loi de Student à $(\mathrm{N}-1)$ degrés de liberté.

$\Phi($.$) est la fonction de répartition de la loi normale$ centrée réduite. 